\title{
Optimal Control Problem with Necessary Optimality Conditions for the Viscous Dullin-Gottwald-Holm Equation
}

\author{
Jinsoo Hwang \\ Department of Mathematics Education, Daegu University, Jillyang, Gyeongsan, Gyeongbuk 712-714, Republic of Korea \\ Correspondence should be addressed to Jinsoo Hwang; jshwang@daegu.ac.kr
}

Received 20 September 2013; Revised 5 January 2014; Accepted 7 January 2014; Published 24 February 2014

Academic Editor: Ziemowit Popowicz

Copyright (C) 2014 Jinsoo Hwang. This is an open access article distributed under the Creative Commons Attribution License, which permits unrestricted use, distribution, and reproduction in any medium, provided the original work is properly cited.

\begin{abstract}
We study the quadratic cost optimal control problems for the viscous Dullin-Gottwald-Holm equation. The main novelty of this paper is to derive the necessary optimality conditions of optimal controls, corresponding to physically meaningful distributive observations. For this we prove the Gâteaux differentiability of nonlinear solution mapping on control variables. Moreover by making use of the second order Gâteaux differentiability of solution mapping on control variables, we prove the local uniqueness of optimal control. This is another novelty of the paper.
\end{abstract}

\section{Introduction}

Recently, in the study of shallow water wave, Dullin et al. [1] derived a new integrable shallow water wave equation with linear and nonlinear dispersion as follows:

$$
y_{t}+2 \omega y_{x}+3 y y_{x}-\alpha^{2}\left(y_{x x t}+2 y_{x} y_{x x}+y y_{x x x}\right)+\gamma y_{x x x}=0 \text {, }
$$

where $y$ is fluid velocity, $\alpha^{2}$ and $\gamma / 2 \omega$ are squares of length scales, and $2 \omega=\sqrt{g h}$ is the linear wave speed for undisturbed water at rest at spatial infinity, where $y$ and its spatial derivatives are taken to vanish. Letting $\alpha^{2} \rightarrow 0$, (1) reduces to the well-known Korteweg-de Vries (KDV) equation (linear dispersion case). And when letting $\gamma \rightarrow 0$, (1) reduces to the Camassa-Holm equation of [2] (nonlinear dispersion case). Usually we can rewrite (1) into

$$
m_{t}+2 \omega y_{x}+y m_{x}+2 y_{x} m+\gamma y_{x x x}=0,
$$

where $m=y-\alpha^{2} y_{x x}$ is a momentum variable. Physically, the third and fourth terms of the left side of (2) represent convection and stretching effects of unidirectional propagation of shallow water waves over a flat bottom, respectively (see $[2,3])$.
Many researchers studied the well-posedness of Cauchy problem for the DGH equation including various properties of the solution of it (see [4-6]).

Recently, Shen et al. [7] studied the optimal control problem of the following viscous DGH equation (cf. [3]):

$$
m_{t}+2 \omega y_{x}+y m_{x}+2 y_{x} m+\gamma y_{x x x}=\nu m_{x x},
$$

where $m=y-y_{x x}$ and $v>0$ stands for the viscosity constant of shallow water wave. As explained in Holm and Staley [8], the small viscosity makes sense to take into account the balance or relaxation between convection and stretching dynamics of shallow water wave.

In [7] Shen et al. studied the distributive optimal control problems of (3) (cf. [3]). For this purpose, they modified (3) to Dirichlet boundary value problem in short interval and proved the existence and uniqueness of $m$ in (3) by weak formulation. However the well-posedness of (3) with respect to $y$ is unclear and the proof contained in [7] relies on the size of $v$ which is an extra condition. Further, in [7] they employed the quadratic cost objective functional to be minimized within an admissible control set with the distributive observation of $m$ in (3) and only discussed the existence of optimal controls which minimize the quadratic cost. But the necessary optimality conditions of optimal controls have not been studied there. 
As for the necessary optimality condition of optimal controls, we can find a recent paper Sun [9]. By employing the Dubovitskii and Milyutin functional analytic approach, Sun has established in [9, Theorem 3] the Pontryagin maximum principle of the optimal control for the viscous DGH equation with the quite general cost which depends on $m$ and not on $y$. Meanwhile, in this paper, we propose the quadratic cost functional for $y$, which is actually more reasonable than that for $m$, and establish the necessary optimality conditions of optimal controls due to Lions [10] in Theorems 5 and 7 for the physically meaningful observations $z=y(u)$ and $z=$ $m(u)$, respectively. To this end, we successfully characterize the Gâteaux derivative $D y(u)(v-u)$ of $y(v)$ in the direction $v-u \in \mathcal{U}$, where $\mathcal{U}$ is a Hilbert space of control variables and $u$ is an optimal control for quadratic cost.

Actually, the extension of optimal control theory to quasilinear equations is not easy. Some researches have been devoted to the study of optimal control or identification problems in specific quasilinear equations. For instance, we can refer to Hwang and Nakagiri $[11,12]$ and Hwang $[13,14]$.

The aim of this paper can be summarized as follows. Firstly, we clarify the well-posedness of (3) with respect to $y$ in the Hadamard sense with appropriate initial value condition in short interval as posed in [7]. Secondly, based on the well-posedness result, we expand the optimal control theory due to Lions [10] with emphasis on deriving necessary optimality conditions of optimal controls in the following distributive control system:

$$
\begin{gathered}
m_{t}(v)-v m_{x x}(v)+2 \omega y_{x}(v)+2 y_{x}(v) m(v) \\
+y(v) m_{x}(v)+\gamma y_{x x x}(v) \\
=f+B v \quad \text { in }(0, T) \times(0,1), \\
y(v ; 0, t)=y(v ; 1, t)=y_{x}(v ; 0, t) \\
=y_{x}(v ; 1, t)=y_{x x}(v ; 0, t) \\
=y_{x x}(v ; 1, t)=0, \quad t \in[0, T], \\
m(v ; x, 0)=m_{0}=y_{0}-y_{0, x x} \quad \text { in }(0,1),
\end{gathered}
$$

where $m(v)=y(v)-y_{x x}(v), f$ is a forcing term, $B$ is a controller, $v$ is a control, and $y(v)$ denotes the state for a given $v \in \mathcal{U}$.

In order to apply the variational approach due to Lions [10] to our problem, we propose the quadratic cost functional $J(v)$ as studied in Lions [10] which is to be minimized within $\mathcal{U}_{\mathrm{ad}} ; \mathcal{U}_{\mathrm{ad}}$ is an admissible set of control variables in $\mathcal{U}$. We show the existence of $u \in \mathscr{U}_{\text {ad }}$ which minimizes the quadratic cost functional $J(v)$. Then, we establish the necessary conditions of optimality of the optimal control $u$ for some physically meaningful observation cases employing the associate adjoint systems. For this we successfully prove the Gâteaux differentiability of the nonlinear solution mapping $v \rightarrow y(v)$, which is used to define the associate adjoint systems.

Moreover, in this paper we discuss the local uniqueness of optimal control. As widely known, it is unclear and difficult to verify the uniqueness of optimal control in nonlinear control problems. By employing the idea given in Ryu [15], we show the strict convexity of the quadratic cost $J(v)$ in local time interval by utilizing the second order Gâteaux differentiability of the nonlinear solution mapping $v \rightarrow y(v)$. Whence by proving strict convexity of the quadratic cost with respect to the control variable, we prove the local uniqueness of optimal control. This is another novelty of the paper.

\section{Preliminaries}

For fixed $T>0$, we set $\Omega=(0,1)$ and $Q=\Omega \times(0, T)$. The scalar products and norms on $L^{2}(\Omega)$ and $H_{0}^{k}(\Omega), k=$ $1,2,3$, are denoted by $(\cdot, \cdot)_{2},|\cdot|_{2}$ and $((\cdot, \cdot))_{k},\|\cdot\|_{k}, k=$ $1,2,3$, respectively. Then, by virtue of Poincare inequality, we can replace these scalar products and norms by $((\cdot, \cdot))_{k}=$ $\left(\partial_{x}^{k}, \partial_{x}^{k} \cdot\right)_{2}$ and $\|\cdot\|_{k}=\left|\partial_{x}^{k} \cdot\right|_{2}, k=1,2,3$, respectively. Let us denote the topological dual spaces of $H_{0}^{k}(\Omega), k=1,2,3$, by $H^{-k}(\Omega), k=1,2,3$. We denote their duality pairing between $H_{0}^{k}(\Omega)$ and $H^{-k}(\Omega)$ by $\langle\cdot, \cdot\rangle_{k,-k}, k=1,2,3$.

We consider the following Dirichlet boundary value problem for the viscous Dullin-Gottwald-Holm (DGH) equation:

$$
\begin{aligned}
& m_{t}-v m_{x x}+2 \omega y_{x}+2 y_{x} m+y m_{x}+\gamma y_{x x x}=f \text { in } Q \text {, } \\
& y(0, t)=y(1, t)=y_{x}(0, t)=y_{x}(1, t) \\
& =y_{x x}(0, t)=y_{x x}(1, t)=0, \quad t \in[0, T], \\
& m(x, 0)=m_{0}=y_{0}-y_{0, x x} \quad \text { in } \Omega \text {, }
\end{aligned}
$$

where $m=y-y_{x x}, f$ is a forcing function, and $m_{0}$ is an initial value.

In order to define weak solutions of (5), we define some Hilbert spaces. At first, $\mathcal{S}(0, T)$ is defined by

$$
\begin{array}{r}
\mathcal{S}(0, T)=\left\{\phi \mid \phi \in L^{2}\left(0, T ; H_{0}^{3}(\Omega)\right),\right. \\
\left.\phi^{\prime} \in L^{2}\left(0, T ; H_{0}^{1}(\Omega)\right)\right\}
\end{array}
$$

endowed with the norm

$$
\|\phi\|_{\mathcal{\delta}(0, T)}=\left(\|\phi\|_{L^{2}\left(0, T ; H_{0}^{3}(\Omega)\right)}^{2}+\left\|\phi^{\prime}\right\|_{L^{2}\left(0, T ; H_{0}^{1}(\Omega)\right)}^{2}\right)^{1 / 2},
$$

where $\phi^{\prime}$ denotes the first order distributional derivatives of $\phi$. Further, $\mathscr{W}(0, T)$ is defined by

$$
\begin{array}{r}
\mathscr{W}(0, T)=\left\{\psi \mid \psi \in L^{2}\left(0, T ; H_{0}^{1}(\Omega)\right),\right. \\
\left.\psi^{\prime} \in L^{2}\left(0, T ; H^{-1}(\Omega)\right)\right\}
\end{array}
$$

endowed with the norm

$$
\|\psi\|_{\mathscr{W}(0, T)}=\left(\|\psi\|_{L^{2}\left(0, T ; H_{0}^{1}(\Omega)\right)}^{2}+\left\|\psi^{\prime}\right\|_{L^{2}\left(0, T ; H^{-1}(\Omega)\right)}^{2}\right)^{1 / 2},
$$

where $\psi^{\prime}$ denotes the first order distributional derivatives of $\psi$. We note here that $\delta(0, T)$ and $\mathscr{W}(0, T)$ are continuously 
imbedded in $C\left([0, T] ; H_{0}^{2}(\Omega)\right)$ and $C\left([0, T] ; L^{2}(\Omega)\right)$, respectively (cf. Dautray and Lions [16, page 555]).

From now on, we will omit writing the integral variables in the definite integral without any confusion.

Lemma 1. Let $\phi$ satisfy the boundary conditions of (5) and $\phi-\phi_{x x} \in \mathscr{W}(0, T)$. Then one has

$$
\|\phi\|_{\mathcal{\delta}(0, T)} \leq C\left\|\phi-\phi_{x x}\right\|_{\mathscr{W}(0, T)},
$$

where $C>0$ is a constant.

Proof. According to the boundary conditions of $\phi$, we have

$$
\begin{aligned}
\left\|\phi-\phi_{x x}\right\|_{\mathscr{W}(0, T)}^{2}= & \int_{0}^{T}\left|\phi_{x}-\phi_{x x x}\right|_{2}^{2} d t \\
& +\int_{0}^{T}\left\|\phi_{t}-\phi_{x x t}\right\|_{H^{-1}(\Omega)}^{2} d t \\
= & \int_{0}^{T}\left(\left|\phi_{x}\right|_{2}^{2}+2\left|\phi_{x x}\right|_{2}^{2}+\left|\phi_{x x x}\right|_{2}^{2}\right) d t \\
& +\int_{0}^{T}\left\|\phi_{t}-\phi_{x x t}\right\|_{H^{-1}(\Omega)}^{2} d t .
\end{aligned}
$$

Since $I-\partial_{x}^{2}: H_{0}^{1}(\Omega) \rightarrow H^{-1}(\Omega)$ is an isomorphism, we can deduce that

R.H.S. of (11)

$$
\begin{aligned}
& \geq \int_{0}^{T}\left|\phi_{x x x}\right|_{2}^{2} d t+c_{0} \int_{0}^{T}\left|\phi_{t x}\right|_{2}^{2} d t \\
& \geq \min \left\{1, c_{0}\right\}\left(\|\phi\|_{L^{2}\left(0, T ; H_{0}^{3}(\Omega)\right)}^{2}+\left\|\phi^{\prime}\right\|_{L^{2}\left(0, T ; H_{0}^{1}(\Omega)\right)}^{2}\right) \\
& =c_{1}\|\phi\|_{\delta(0, T)}^{2},
\end{aligned}
$$

where $c_{0}, c_{1}>0$ are constants. Thus we prove this lemma.

The following variational formulation is used to define the weak solution of (5).

Definition 2. A function $y \in \mathcal{S}(0, T)$ is said to be a weak solution of (5) if $m=y-y_{x x} \in \mathscr{W}(0, T)$ and $m=y-y_{x x}$ satisfies

$$
\begin{aligned}
\left\langle m^{\prime}(\cdot), \phi\right\rangle_{-1,1}+\nu\left(m_{x}(\cdot), \phi_{x}\right)_{2} \\
\quad+2 \omega\left(y_{x}(\cdot), \phi\right)_{2}+2\left(y_{x} m, \phi\right)_{2} \\
\quad+\left(y(\cdot) m_{x}(\cdot), \phi\right)_{2}+\gamma\left(y_{x x x}(\cdot), \phi\right)_{2} \\
=\langle f(\cdot), \phi\rangle_{-1,1}
\end{aligned}
$$

for all $\phi \in H_{0}^{1}(\Omega)$ in the sense of $\mathscr{D}^{\prime}(0, T)$,

$m(0)=m_{0}=y_{0}-y_{0, x x}$.

In order to verify the well-posedness of (5), we partially refer to the result by Shen et al. [7]. The well-posedness of (5) in the sense of Hadamard can be given as follows.
Theorem 3. Assume that $v>0, f \in L^{2}\left(0, T ; H^{-1}(\Omega)\right)$, and $y_{0} \in H_{0}^{2}(\Omega)$. Then the problem (5) has a unique solution $y$ in $\delta(0, T)$. And the solution mapping $p=\left(y_{0}, f\right) \rightarrow y(p)$ of $P \equiv H_{0}^{2}(\Omega) \times L^{2}\left(0, T ; H^{-1}(\Omega)\right)$ into $\mathcal{S}(0, T)$ is a local Lipschitz continuous; that is, for each $p_{1}=\left(y_{0}^{1}, f_{1}\right) \in P$ and $p_{2}=\left(y_{0}^{2}, f_{2}\right) \in P$, one has the inequality

$$
\begin{aligned}
& \left\|y_{1}\left(p_{1}\right)-y_{2}\left(p_{2}\right)\right\|_{\mathcal{\delta}(0, T)} \\
& \quad \leq C\left(\left\|y_{0}^{1}-y_{0}^{2}\right\|_{H_{0}^{2}(\Omega)}+\left\|f_{1}-f_{2}\right\|_{L^{2}\left(0, T ; H^{-1}(\Omega)\right)}\right),
\end{aligned}
$$

where $C$ is a constant which depends on $y_{1}$ and $y_{2}$.

Remark 4. In [7], the well-posedness of (5) is partially verified, which is indeed the case that the viscosity constant $v>0$ is large enough. However, as we will see in the Appendix, such an extra assumption can be removed.

Proof of Theorem 3. By utilizing the result of [7], combined with Lemma 1, we can know that (5) possesses a unique solution $y \in \mathcal{S}(0, T)$ under the data condition $p=\left(y_{0}, f\right) \in$ $H_{0}^{2}(\Omega) \times L^{2}\left(0, T ; H^{-1}(\Omega)\right)$.

Based on the above result, we prove the inequality (14). For that purpose, we denote $y_{1}-y_{2} \equiv y\left(p_{1}\right)-y\left(p_{2}\right)$ by $\psi$ and $y_{i}-y_{i, x x}$ by $m_{i}, i=1,2$. Then, we can observe from (5) that

$$
\begin{gathered}
\Psi_{t}-\nu \Psi_{x x}+2 \omega \psi_{x}+2 \psi_{x} m_{1}+2 y_{2, x} \Psi \\
+\psi m_{1, x}+y_{2} \Psi_{x}+\gamma \psi_{x x x}=f_{1}-f_{2} \quad \text { in } Q, \\
\psi(0, t)=\psi(1, t)=\psi_{x}(0, t)=\psi_{x}(1, t)=\psi_{x x}(0, t) \\
=\psi_{x x}(1, t)=0, \quad t \in[0, T], \\
\Psi(x, 0)=\Psi_{0}=m_{0}^{1}-m_{0}^{2} \quad \text { in } \Omega,
\end{gathered}
$$

where $\Psi=\psi-\psi_{x x}$ and $m_{0}^{i}=y_{0}^{i}-y_{0, x x}^{i}, i=1,2$. Multiplying $\Psi$ in both sides of (15), we have

$$
\begin{aligned}
\frac{1}{2} \frac{d}{d t}|\Psi|_{2}^{2}+\nu\left|\Psi_{x}\right|_{2}^{2}= & -2 \omega\left(\psi_{x}, \Psi\right)_{2} \\
& -\left(2 \psi_{x} m_{1}, \Psi\right)_{2}-\left(2 y_{2, x} \Psi, \Psi\right)_{2} \\
& -\left(\psi m_{1, x}, \Psi\right)_{2}-\left(y_{2} \Psi_{x}, \Psi\right)_{2} \\
& -\gamma\left(\psi_{x x x}, \Psi\right)_{2}+\left\langle f_{1}-f_{2}, \Psi\right\rangle_{-1,1} .
\end{aligned}
$$

And we integrate $(16)$ over $[0, t]$ to have

$$
\begin{aligned}
\frac{1}{2}|\Psi(t)|_{2}^{2}+v \int_{0}^{t}\left|\Psi_{x}\right|_{2}^{2} d s \\
=\frac{1}{2}\left|\Psi_{0}\right|_{2}^{2}-2 \omega \int_{0}^{t}\left(\psi_{x}, \Psi\right)_{2} d s-\int_{0}^{t}\left(2 \psi_{x} m_{1}, \Psi\right)_{2} d s \\
\quad-\int_{0}^{t}\left(2 y_{2, x} \Psi, \Psi\right)_{2} d s-\int_{0}^{t}\left(\psi m_{1, x}, \Psi\right)_{2} d s \\
\quad-\int_{0}^{t}\left(y_{2} \Psi_{x}, \Psi\right)_{2} d s-\gamma \int_{0}^{t}\left(\psi_{x x x}, \Psi\right)_{2} d s \\
+\int_{0}^{t}\left\langle f_{1}-f_{2}, \Psi\right\rangle_{-1,1} d s .
\end{aligned}
$$


4

Abstract and Applied Analysis

By Sobolev embedding theorem, $m_{i} \in \mathscr{W}(0, T) \hookrightarrow$ $C\left([0, T] ; L^{2}(\Omega)\right), i=1,2$, and $|\Psi|_{2}^{2}=|\psi|_{2}^{2}+2\left|\psi_{x}\right|_{2}^{2}+\left|\psi_{x x}\right|_{2}^{2}$, the right members of (17) can be estimated as follows:

$$
\begin{aligned}
& \left|-2 \omega \int_{0}^{t}\left(\psi_{x}, \Psi\right)_{2} d s\right| \\
& \leq 2 \omega \int_{0}^{t}\left|\psi_{x}\right|_{2}|\Psi|_{2} d s \\
& \leq 2 \omega \int_{0}^{t}|\Psi|_{2}^{2} d s \\
& \left|-\int_{0}^{t}\left(2 \psi_{x} m_{1}, \Psi\right)_{2} d s\right| \\
& \leq 2 \int_{0}^{t}\left\|\psi_{x}\right\|_{L^{\infty}(\Omega)}\left|m_{1}\right|_{2}|\Psi|_{2} d s \\
& \leq c_{0}\left\|m_{1}\right\|_{C\left([0, T] ; L^{2}(\Omega)\right)} \int_{0}^{t}\left|\psi_{x x}\right|_{2}|\Psi|_{2} d s \\
& \leq c_{1} \int_{0}^{t}|\Psi|_{2}^{2} d s \\
& \left|-\int_{0}^{t}\left(2 y_{2, x} \Psi, \Psi\right)_{2} d s\right| \\
& \leq 2\left\|y_{2, x}\right\|_{L^{\infty}(\mathrm{Q})} \int_{0}^{t}|\Psi|_{2}^{2} d s \\
& \leq c_{2} \int_{0}^{t}|\Psi|_{2}^{2} d s \\
& \left|-\int_{0}^{t}\left(\psi m_{1, x}, \Psi\right)_{2} d s\right| \\
& \leq \int_{0}^{t}\|\psi\|_{L^{\infty}(\Omega)}\left|m_{1, x}\right|_{2}|\Psi|_{2} d s \\
& \leq c_{3} \int_{0}^{t}\left|\psi_{x}\right|_{2}\left|m_{1, x}\right|_{2}|\Psi|_{2} d s \\
& \leq c_{4} \int_{0}^{t}\left|m_{1, x}\right|_{2}|\Psi|_{2}^{2} d s ; \\
& \left|-\int_{0}^{t}\left(y_{2} \Psi_{x}, \Psi\right)_{2} d s\right| \\
& \leq\left\|y_{2}\right\|_{L^{\infty}(\mathrm{Q})} \int_{0}^{t}\left|\Psi_{x}\right|_{2}|\Psi|_{2} d s \\
& \leq c_{5} \int_{0}^{t}|\Psi|_{2}^{2} d s+\frac{v}{6} \int_{0}^{t}\left|\Psi_{x}\right|_{2}^{2} d s ; \\
& \left|-\gamma \int_{0}^{t}\left(\psi_{x x x}, \Psi\right)_{2} d s\right| \\
& =\left|\gamma \int_{0}^{t}\left(\psi_{x x}, \Psi_{x}\right)_{2} d s\right| \\
& \leq \gamma \int_{0}^{t}\left|\psi_{x x}\right|_{2}\left|\Psi_{x}\right|_{2} d s
\end{aligned}
$$

$$
\begin{aligned}
& \leq \gamma \int_{0}^{t}|\Psi|_{2}\left|\Psi_{x}\right|_{2} d s \\
& \leq c_{6} \int_{0}^{t}|\Psi|_{2}^{2} d s+\frac{\nu}{6} \int_{0}^{t}\left|\Psi_{x}\right|_{2}^{2} d s \\
&\left|\int_{0}^{t}\left\langle f_{1}-f_{2}, \Psi\right\rangle_{-1,1} d s\right| \\
& \leq \int_{0}^{t}\left\|f_{1}-f_{2}\right\|_{H^{-1}(\Omega)}\left|\Psi_{x}\right|_{2} d s \\
& \leq c_{7}\left\|f_{1}-f_{2}\right\|_{L^{2}\left(0, T ; H^{-1}(\Omega)\right)}^{2} \\
&+\frac{\nu}{6} \int_{0}^{t}\left|\Psi_{x}\right|_{2}^{2} d s,
\end{aligned}
$$

where $c_{0}, \ldots, c_{7}$ are constants. We replace the right hand side of (17) by the right members of (18). Then we have

$$
\begin{aligned}
& |\Psi(t)|_{2}^{2}+\int_{0}^{t}\left|\Psi_{x}\right|_{2}^{2} d s \\
& \quad \leq c_{8}\left(\left|\Psi_{0}\right|_{2}^{2}+\left\|f_{1}-f_{2}\right\|_{L^{2}\left(0, T ; H^{-1}(\Omega)\right)}^{2}+\int_{0}^{t}\left|m_{1, x}\right|_{2}|\Psi|_{2}^{2} d s\right),
\end{aligned}
$$

where $c_{8}$ is a constant, depending on $y_{1}$ and $y_{2}$. And we apply the Gronwall inequality to (19) to obtain

$$
\begin{aligned}
|\Psi(t)|_{2}^{2}+\int_{0}^{t}\left|\Psi_{x}\right|_{2}^{2} d s \\
\leq c_{8}\left(\left|\Psi_{0}\right|_{2}^{2}+\left\|f_{1}-f_{2}\right\|_{L^{2}\left(0, T ; H^{-1}(\Omega)\right)}^{2}\right) \\
\quad \times \exp \left(c_{8}\left\|m_{1}\right\|_{L^{1}\left(0, T ; H_{0}^{1}(\Omega)\right)}\right) \\
\leq c_{9}\left(\left|\Psi_{0}\right|_{2}^{2}+\left\|f_{1}-f_{2}\right\|_{L^{2}\left(0, T ; H^{-1}(\Omega)\right)}^{2}\right),
\end{aligned}
$$

where $c_{9}$ is a constant, depending on $y_{1}$ and $y_{2}$. Next we estmate $\Psi_{t}$ in (15) as follows:

$$
\begin{aligned}
\left\|\Psi_{t}\right\|_{H^{-1}(\Omega)} \leq & v\left|\Psi_{x}\right|_{2}+2 \omega\left|\psi_{x}\right|_{2}+2\left\|m_{1}\right\|_{L^{\infty}(\Omega)}\left|\psi_{x}\right|_{2} \\
& +2\left\|y_{2, x}\right\|_{L^{\infty}(Q)}|\Psi|_{2} \\
& +\|\psi\|_{L^{\infty}(\Omega)}\left|m_{1, x}\right|_{2}+\left\|y_{2}\right\|_{L^{\infty}(Q)}\left|\Psi_{x}\right|_{2} \\
& +\gamma\left|\psi_{x x x}\right|_{2}+\left\|f_{1}-f_{2}\right\|_{H^{-1}(\Omega)} .
\end{aligned}
$$

By Sobolev embedding theorem, we have the following inequality:

$$
\begin{aligned}
\text { R.H.S. of }(21) \leq & c_{10}\left|\Psi_{x}\right|_{2}+c_{11}\left(\left|m_{1, x}\right|_{2}+1\right)|\Psi|_{2} \\
& +\left\|f_{1}-f_{2}\right\|_{H^{-1}(\Omega)},
\end{aligned}
$$


where $c_{10}, c_{11}$ are constants, depending on $y_{1}$ and $y_{2}$. By (21) and (22) we can obtain

$$
\begin{aligned}
& \left\|\Psi_{t}\right\|_{L^{2}\left(0, T ; H^{-1}(\Omega)\right)}^{2} \\
& \leq c_{12}\left(\|\Psi\|_{L^{2}\left(0, T ; H_{0}^{1}(\Omega)\right)}^{2}+\left(\left\|m_{1}\right\|_{L^{2}\left(0, T ; H_{0}^{1}(\Omega)\right)}^{2}+1\right)\right. \\
& \left.\quad \times\|\Psi\|_{L^{\infty}\left(0, T ; L^{2}(\Omega)\right)}^{2}+\left\|f_{1}-f_{2}\right\|_{L^{2}\left(0, T ; H^{-1}(\Omega)\right)}^{2}\right) \\
& \leq c_{13}\left(\left|\Psi_{0}\right|_{2}^{2}+\left\|f_{1}-f_{2}\right\|_{L^{2}\left(0, T ; H^{-1}(\Omega)\right)}^{2}\right),
\end{aligned}
$$

where $c_{12}, c_{13}$ are constants, depending on $y_{1}$ and $y_{2}$. We can deduce that (20) and (23) imply

$$
\|\Psi\|_{\mathscr{W}(0, T)} \leq c_{14}\left(\left|\Psi_{0}\right|_{2}+\left\|f_{1}-f_{2}\right\|_{L^{2}\left(0, T ; H^{-1}(\Omega)\right)}\right),
$$

where $c_{14}$ is a constant, depending on $y_{1}$ and $y_{2}$. Finally from Lemma 1 and (24) we have

$$
\begin{aligned}
\|\psi\|_{\delta(0, T)} & \equiv\left\|y_{1}\left(p_{1}\right)-y_{2}\left(p_{2}\right)\right\|_{\mathcal{\delta}(0, T)} \\
& \leq c_{15}\left(\left|\Psi_{0}\right|_{2}+\left\|f_{1}-f_{2}\right\|_{L^{2}\left(0, T ; H^{-1}(\Omega)\right)}\right) \\
& \leq C\left(\left\|y_{0}^{1}-y_{0}^{2}\right\|_{H_{0}^{2}(\Omega)}+\left\|f_{1}-f_{2}\right\|_{L^{2}\left(0, T ; H^{-1}(\Omega)\right)}\right),
\end{aligned}
$$

where $c_{15}, C$ are constants, depending on $y_{1}$ and $y_{2}$. Thus we complete the proof.

\section{Quadratic Cost Optimal Control Problems}

In this section we study the quadratic cost optimal control problems for the viscous DGH equation due to the theory of Lions [10]. Let $\mathscr{U}$ be a Hilbert space of control variables, and let $B$ be an operator,

$$
B \in \mathscr{L}\left(\mathscr{U}, L^{2}\left(0, T ; L^{2}(\Omega)\right)\right),
$$

called a controller. We consider the following nonlinear control system:

$$
\begin{gathered}
m_{t}(v)-v m_{x x}(v)+2 \omega y_{x}(v)+2 y_{x}(v) m(v) \\
+y(v) m_{x}(v)+\gamma y_{x x x}(v)=f+B v \quad \text { in } Q, \\
y(v ; 0, t)=y(v ; 1, t)=y_{x}(v ; 0, t) \\
=y_{x}(v ; 1, t)=y_{x x}(v ; 0, t) \\
=y_{x x}(v ; 1, t)=0, \quad t \in[0, T], \\
m(v ; x, 0)=m_{0}=y_{0}-y_{0, x x}, \quad \text { in } \Omega,
\end{gathered}
$$

where $m(v)=y(v)-y_{x x}(v), m_{0} \in L^{2}(\Omega), f \in L^{2}\left(0, T ; L^{2}(\Omega)\right)$, and $v \in \mathcal{U}$ is a control. By virtue of Theorem 3 and (26), we can define uniquely the solution map $v \rightarrow y(v)$ of $\mathcal{U}$ into $\delta(0, T)$. We will call the solution $y(v)$ of $(27)$ the state of the control system (27). The observation of the state is assumed to be given by

$$
z(v)=C y(v), \quad C \in \mathscr{L}(\mathcal{S}(0, T), M),
$$

where $C$ is an operator called the observer and $M$ is a Hilbert space of observation variables. The quadratic cost function associated with the control system (27) is given by

$$
J(v)=\left\|C y(v)-Y_{d}\right\|_{M}^{2}+(R v, v)_{\mathscr{U}} \quad \text { for } v \in \mathcal{U},
$$

where $Y_{d} \in M$ is a desired value of $y(v)$ and $R \in \mathscr{L}(\mathscr{U}, \mathscr{U})$ is symmetric and positive; that is,

$$
(R v, v)_{\mathscr{U}}=(v, R v)_{\mathscr{U}} \geq d\|v\|_{\mathscr{U}}^{2}
$$

for some $d>0$. Let $\mathscr{U}_{\text {ad }}$ be a closed convex subset of $\mathcal{U}$, which is called the admissible set. An element $u \in \mathcal{U}_{\text {ad }}$ which attains the minimum of $J(v)$ over $\mathscr{U}_{\text {ad }}$ is called an optimal control for the cost (29).

In this section we will characterize the optimal controls by giving necessary conditions for optimality. For this it is necessary to write down the necessary optimality condition,

$$
D J(u)(v-u) \geq 0 \text { for all } v \in \mathscr{U}_{\mathrm{ad}},
$$

and to analyze (31) in view of the proper adjoint state system, where $D J(u)$ denote the Gâteaux derivative of $J(v)$ at $v=u$. And we study local uniqueness of the optimal control.

As indicated in Section 1, we show the existence of an optimal control and give the characterizations of them.

3.1. Existence of the Optimal Control. Now we show the existence of an optimal control $u$ for the cost (29).

Theorem 5. Assume that the hypotheses of Theorem 3 are satisfied. Then there exists at least one optimal control $u$ for the control problem (27) with the cost (29).

Proof. Set $J=\inf _{v \in \mathscr{U}_{\mathrm{ad}}} J(v)$. Since $\mathscr{U}_{\mathrm{ad}}$ is nonempty, there is a sequence $\left\{v_{n}\right\}$ in $\mathscr{U}$ such that

$$
\inf _{v \in \mathscr{U}_{\mathrm{ad}}} J(v)=\lim _{n \rightarrow \infty} J\left(v_{n}\right)=J .
$$

Obviously $\left\{J\left(v_{n}\right)\right\}$ is bounded in $\mathbf{R}^{+}$. Then by (30) there exists a constant $K_{0}>0$ such that

$$
d\left\|v_{n}\right\|_{\mathscr{U}}^{2} \leq\left(R v_{n}, v_{n}\right)_{\mathscr{U}} \leq J\left(v_{n}\right) \leq K_{0} .
$$

This shows that $\left\{v_{n}\right\}$ is bounded in $\mathcal{U}$. Since $\mathscr{U}_{\text {ad }}$ is closed and convex, we can choose a subsequence (denoted again by $\left\{v_{n}\right\}$ ) of $\left\{v_{n}\right\}$ and find $a u \in \mathscr{U}_{\text {ad }}$ such that

$$
v_{n} \longrightarrow u \text { weakly in } \mathcal{U}
$$

as $n \rightarrow \infty$. From now on, each state $y_{n}=y\left(v_{n}\right) \in \delta(0, T)$ corresponding to $v_{n}$ is the solution of

$$
\begin{gathered}
m_{n, t}-v m_{n, x x}+2 \omega y_{n, x}+2 y_{n, x} m_{n} \\
+y_{n} m_{n, x}+\gamma y_{n, x x x}=f+B v_{n} \quad \text { in } Q, \\
y_{n}(0, t)=y_{n}(1, t)=y_{n, x}(0, t)=y_{n, x}(1, t)=y_{n, x x}(0, t) \\
=y_{n, x x}(1, t)=0, \quad t \in[0, T], \\
m_{n}(x, 0)=m_{0}=y_{0}-y_{0, x x} \quad \text { in } \Omega,
\end{gathered}
$$


where $m_{n}=y_{n}-y_{n, x x}$. By (33) the term $B v_{n}$ is estimated as

$$
\begin{aligned}
\left\|B v_{n}\right\|_{L^{2}\left(0, T ; L^{2}(\Omega)\right)} & \leq\|B\|_{\mathscr{L}\left(\mathcal{U}, L^{2}\left(0, T ; L^{2}(\Omega)\right)\right)}\left\|v_{n}\right\|_{\mathscr{U}} \\
& \leq\|B\|_{\mathscr{L}\left(\mathcal{U}, L^{2}\left(0, T ; L^{2}(\Omega)\right)\right)} \sqrt{K_{0} d^{-1}} \equiv K_{1} .
\end{aligned}
$$

Hence we can deduce from Theorem 3 that

$$
\left\|m_{n}\right\|_{\mathscr{W}(0, T)} \leq C_{0}\left(\left\|y_{0}\right\|_{H_{0}^{2}(\Omega)}+\|f\|_{L^{2}\left(0, T ; L^{2}(\Omega)\right)}+K_{1}\right)
$$

for some $C_{0}>0$. And also from Lemma 1 we can know that

$$
\left\|y_{n}\right\|_{\delta(0, T)} \leq C_{1}\left(\left\|y_{0}\right\|_{H_{0}^{2}(\Omega)}+\|f\|_{L^{2}\left(0, T ; L^{2}(\Omega)\right)}+K_{1}\right)
$$

for some $C_{1}>0$. Therefore, by the extraction theorem of Rellich's, we can find a subsequence of $\left\{m_{n}\right\}$, say again $\left\{m_{n}\right\}$, and find a $m=y-y_{x x} \in \mathscr{W}(0, T)$ such that

$$
m_{n} \longrightarrow m \text { weakly in } \mathscr{W}(0, T) \text {. }
$$

By using the fact that $H_{0}^{1}(\Omega) \hookrightarrow L^{2}(\Omega)$ is compact and by virtue of (39), we can refer to the result of the Aubin-LionsTemam's compact imbedding theorem (cf. Temam [17, page 271]) to verify that $\left\{m_{n}\right\}$ is precompact in $L^{2}\left(0, T ; L^{2}(\Omega)\right)$. Hence there exists a subsequence $\left\{m_{n_{k}}\right\} \subset\left\{m_{n}\right\}$ such that

$$
m_{n_{k}} \longrightarrow m \text { strongly in } L^{2}\left(0, T ; L^{2}(\Omega)\right) \text { as } k \longrightarrow \infty \text {. }
$$

Since $m_{n}=y_{n}-y_{n, x x} \in \mathscr{W}(0, T) \hookrightarrow C\left([0, T] ; L^{2}(\Omega)\right)$, we know that $y_{n} \in C\left([0, T] ; H_{0}^{2}(\Omega)\right)$. And from (40) we can choose a subsequence of $\left\{y_{n_{k}}\right\}$, denoted again by $\left\{y_{n_{k}}\right\}$ such that

$$
y_{n_{k}}(t) \longrightarrow y(t) \quad \text { strongly in } H_{0}^{2}(\Omega) \text { for a.e. } t \in[0, T] .
$$

We use (39)-(41) and apply the Lebesgue dominated convergence theorem to have

$$
\begin{aligned}
2 y_{n_{k}, x} m_{n_{k}} & \longrightarrow 2 y_{x} m \quad \text { strongly in } L^{2}\left(0, T ; L^{2}(\Omega)\right), \\
y_{n_{k}} m_{n_{k} x} & \longrightarrow y m_{x} \quad \text { weakly in } L^{2}\left(0, T ; L^{2}(\Omega)\right)
\end{aligned}
$$

as $k \rightarrow \infty$. We replace $y_{n}$ and $m_{n}$ by $y_{n_{k}}$ and $m_{n_{k}}$, respectively, and take $k \rightarrow \infty$ in (35). Then by the standard argument in Dautray and Lions [16, pages 561-565], we conclude that the limit $m$ satisfies

$$
\begin{aligned}
& m_{t}-v m_{x x}+2 \omega y_{x}+2 y_{x} m+y m_{x}+\gamma y_{x x x} \\
&=f+B u \quad \text { in } Q \\
& y(0, t)=y(1, t)=y_{x}(0, t)=y_{x}(1, t)=y_{x x}(0, t) \\
&=y_{x x}(1, t)=0, \quad t \in[0, T], \\
& m(x, 0)=m_{0}=y_{0}-y_{0, x x}, \quad \text { in } \Omega
\end{aligned}
$$

in weak sense, where $m=y-y_{x x}$. Moreover the uniqueness of weak solutions in (43) via Theorem 3 enables us to conclude that $y=y(u)$ in $\delta(0, T)$, which implies $y\left(v_{n}\right) \rightarrow y(u)$ weakly in $\mathcal{S}(0, T)$. Since $C$ is continuous on $\mathcal{S}(0, T)$ and $\|\cdot\|_{M}$ is lower semicontinuous, it follows that

$$
\left\|C y(u)-z_{d}\right\|_{M} \leq \liminf _{n \rightarrow \infty}\left\|C y\left(v_{n}\right)-z_{d}\right\|_{M} .
$$

It is also clear from $\liminf _{k \rightarrow \infty}\left\|\mathrm{R}^{1 / 2} v_{n}\right\|_{\mathscr{U}} \geq\left\|R^{1 / 2} v\right\|_{\mathscr{U}}$ that $\liminf _{k \rightarrow \infty}\left(R v_{n}, v_{n}\right)_{\mathcal{u}} \geq(R u, u)_{\mathcal{U}}$. Hence

$$
J=\liminf _{n \rightarrow \infty} J\left(v_{n}\right) \geq J(u)
$$

But since $J(u) \geq J$ by definition, we conclude that $J(u)=$ $\inf _{v \in \mathcal{U}_{\mathrm{ad}}} J(v)$. This completes the proof.

3.2. Gâteaux Differentiability of Solution Mapping. In order to characterize the optimal control which satisfies the necessary optimality condition (31), we need to prove the Gâteaux differentiability of the mapping $v \rightarrow y(v)$ of $\mathcal{U} \rightarrow \mathcal{S}(0, T)$.

Definition 6. The solution map $v \rightarrow y(v)$ of $\mathcal{U}$ into $\mathcal{S}(0, T)$ is said to be Gâteaux differentiable at $v=u$ if for any $w \in \mathcal{U}$ there exists a $D y(u) \in \mathscr{L}(\mathcal{U}, \mathcal{S}(0, T))$ such that

$$
\left\|\frac{1}{\lambda}(y(u+\lambda w)-y(u))-D y(u) w\right\|_{\mathcal{S}(0, T)} \longrightarrow 0 \text { as } \lambda \longrightarrow 0 .
$$

The operator $D y(u)$ denotes the Gâteaux derivative of $y(u)$ at $v=u$ and the function $D y(u) w \in \delta(0, T)$ is called the Gâteaux derivative in the direction $w \in \mathcal{U}$, which plays an important role in the nonlinear optimal control problem.

Theorem 7. The map $v \rightarrow y(v)$ of $\mathcal{U}$ into $\mathcal{S}(0, T)$ is Gâteaux differentiable at $v=u$ and such the Gâteaux derivative of $y(v)$ at $v=u$ in the direction $v-u \in \mathcal{U}$, say $z=D y(u)(v-u)$, is a unique solution of the following problem:

$$
\begin{gathered}
\mathscr{Z}_{t}-v \mathscr{Z}_{x x}+2 \omega z_{x}+2 z_{x} m+2 y_{x}(u) \mathscr{Z} \\
+z m_{x}+y(u) \mathscr{Z}_{x}+\gamma z_{x x x}=B(v-u) \quad \text { in } Q, \\
z(0, t)=z(1, t)=z_{x}(0, t)=z_{x}(1, t)=z_{x x}(0, t) \\
=z_{x x}(1, t)=0, \quad t \in[0, T], \\
\mathscr{Z}(x, 0)=0 \quad \text { in } \Omega,
\end{gathered}
$$

where $m=y(u)-y_{x x}(u)$ and $\mathscr{Z}=z-z_{x x}$.

Proof. Let $\lambda \in(-1,1), \lambda \neq 0$. We set $w=v-u$ and

$$
z_{\lambda}=\lambda^{-1}(y(u+\lambda w)-y(u)) .
$$

Then $z_{\lambda}$ satisfies

$$
\begin{aligned}
\mathscr{Z}_{\lambda, t} & -v \mathscr{Z}_{\lambda, x x}+2 \omega z_{\lambda, x}+2 z_{\lambda, x} m_{\lambda}+2 y_{x}(u) \mathscr{Z}_{\lambda} \\
& +z_{\lambda} m_{\lambda, x}+y(u) \mathscr{Z}_{\lambda, x} \\
& +\gamma z_{\lambda, x x x}=B w \quad \text { in } Q,
\end{aligned}
$$




$$
\begin{aligned}
& z_{\lambda}(0, t)=z_{\lambda}(1, t)=z_{\lambda, x}(0, t)=z_{\lambda, x}(1, t)=z_{\lambda, x x}(0, t) \\
& =z_{\lambda, x x}(1, t)=0, \quad t \in[0, T], \\
& \mathscr{Z}_{\lambda}(x, 0)=0 \quad \text { in } \Omega,
\end{aligned}
$$

where $m_{\lambda}=y(u+\lambda w)-y_{x x}(u+\lambda w)$ and $\mathscr{E}_{\lambda}=z_{\lambda}-z_{\lambda, x x}$.

By the continuity of (14), we have

$$
\|y(u+\lambda w)-y(u)\|_{\mathcal{\delta}(0, T)} \leq|\lambda| C\|B w\|_{L^{2}\left(0, T ; L^{2}(\Omega)\right)},
$$

where $C$ is a constant, depending on $y(u)$ and $y(u+\lambda w)$. Hence we have

$$
\left\|z_{\lambda}\right\|_{\mathcal{\delta}(0, T)} \leq C\|B w\|_{L^{2}\left(0, T ; L^{2}(\Omega)\right)}<\infty .
$$

Therefore, we can infer that there exists a $z \in \mathcal{S}(0, T)$ and a sequence $\left\{\lambda_{k}\right\} \subset(-1,1)$ tending to 0 such that

$$
z_{\lambda_{k}} \longrightarrow z \quad \text { weakly in } \mathcal{S}(0, T)
$$

as $k \rightarrow \infty$. Since the imbedding $\mathcal{S}(0, T) \hookrightarrow L^{2}\left(0, T ; H_{0}^{2}(\Omega)\right)$ is compact ([17, page 271]), it is implied from (52) that

$$
z_{\lambda_{k}}(t) \longrightarrow z(t) \quad \text { strongly in } H_{0}^{2}(\Omega) \text { a.e. } t \in[0, T]
$$

for some $\left\{\lambda_{k}\right\} \subset(-1,1)$ tending to 0 as $k \rightarrow \infty$. Whence by (50)-(53) and Lebesgue dominated convergence theorem we can easily show that

$$
\begin{aligned}
2 z_{\lambda_{k}, x} m_{\lambda_{k}} & \longrightarrow 2 z_{x} m \quad \text { strongly in } L^{2}\left(0, T ; L^{2}(\Omega)\right), \\
z_{\lambda_{k}} m_{\lambda_{k}, x} & \longrightarrow z m_{x} \quad \text { strongly in } L^{2}\left(0, T ; L^{2}(\Omega)\right), \\
\mathscr{Z}_{\lambda_{k}} & \longrightarrow \mathscr{Z} \quad \text { weakly in } L^{2}\left(0, T ; H_{0}^{1}(\Omega)\right)
\end{aligned}
$$

as $k \rightarrow \infty$, where $m=y(u)-y_{x x}(u)$ and $\mathscr{Z}=z-z_{x x}$. And also we can deduce from (49), (52), and (56) that

$$
\mathscr{Z}_{\lambda_{k}, t} \longrightarrow \mathscr{Z}_{t} \quad \text { weakly in } L^{2}\left(0, T ; H^{-1}(\Omega)\right)
$$

as $k \rightarrow \infty$.

Hence we can see from (52) to (57) that $z_{\lambda} \rightarrow z=$ $D y(u) w$ weakly in $\mathcal{S}(0, T)$ as $\lambda \rightarrow 0$ in which $z$ is a solution of (47). This convergence can be improved by showing the strong convergence of $\left\{z_{\lambda}\right\}$ also in the topology of $\mathcal{S}(0, T)$.

Subtracting (47) from (49) and denoting $z_{\lambda}-z$ by $\phi_{\lambda}$, we obtain that

$$
\begin{aligned}
& \Phi_{\lambda, t}-\nu \Phi_{\lambda, x x}+2 \omega \phi_{\lambda, x}+2 y_{x}(u) \Phi_{\lambda} \\
& \quad+y(u) \Phi_{\lambda, x}+\gamma \phi_{\lambda, x x x}=\epsilon(\lambda) \quad \text { in } Q, \\
& \phi_{\lambda}(0, t)=\phi_{\lambda}(1, t)=\phi_{\lambda, x}(0, t)=\phi_{\lambda, x}(1, t)=\phi_{\lambda, x x}(0, t) \\
& \quad=\phi_{\lambda, x x}(1, t)=0, \quad t \in[0, T], \\
& \Phi_{\lambda}(x, 0)=0 \quad \text { in } \Omega,
\end{aligned}
$$

where $\Phi_{\lambda}=\phi_{\lambda}-\phi_{\lambda, x x}$ and $\epsilon(\lambda)=-2 z_{\lambda, x} m_{\lambda}+2 z_{x} m-z_{\lambda} m_{\lambda, x}+$ $z m_{x}$.

From (54) and (55) we know that

$$
\epsilon(\lambda) \longrightarrow 0 \text { strongly in } L^{2}\left(0, T ; L^{2}(\Omega)\right) \text { as } \lambda \longrightarrow 0 \text {. }
$$

In order to estimate $\phi_{\lambda}$ we multiply $\Phi_{\lambda}$ in both sides of (58) and integrate it over $[0, t]$ to have

$$
\begin{aligned}
\left|\Phi_{\lambda}(t)\right|_{2}^{2} & +2 v \int_{0}^{t}\left|\Phi_{\lambda, x}\right|_{2}^{2} d s \\
= & -2 \int_{0}^{t}\left(2 \omega \phi_{\lambda, x}, \Phi_{\lambda}\right)_{2} d s-2 \int_{0}^{t}\left(2 y_{x}(u) \Phi_{\lambda}, \Phi_{\lambda}\right)_{2} d s \\
& -2 \int_{0}^{t}\left(y(u) \Phi_{\lambda, x}, \Phi_{\lambda}\right)_{2} d s \\
& -2 \int_{0}^{t}\left(\gamma \phi_{\lambda, x x x}, \Phi_{\lambda}\right)_{2} d s+2 \int_{0}^{t}\left(\epsilon(\lambda), \Phi_{\lambda}\right)_{2} d s .
\end{aligned}
$$

The integral parts of the right member of (60) can be estimated as follows:

$$
\begin{aligned}
\left|-2 \int_{0}^{t}\left(2 \omega \phi_{\lambda, x}, \Phi_{\lambda}\right)_{2} d s\right| & \leq 4 \omega \int_{0}^{t}\left|\phi_{\lambda, x}\right|_{2}\left|\Phi_{\lambda}\right|_{2} d s \\
\leq & 4 \omega \int_{0}^{t}\left|\Phi_{\lambda}\right|_{2}^{2} d s ; \\
\left|-2 \int_{0}^{t}\left(2 y_{x}(u) \Phi_{\lambda}, \Phi_{\lambda}\right)_{2} d s\right| & \leq 4\left\|y_{x}(u)\right\|_{L^{\infty}(Q)} \int_{0}^{t}\left|\Phi_{\lambda}\right|_{2}^{2} d s \\
\leq & c_{0} \int_{0}^{t}\left|\Phi_{\lambda}\right|_{2}^{2} d s ; \\
\left|-2 \int_{0}^{t}\left(y(u) \Phi_{\lambda, x}, \Phi_{\lambda}\right)_{2} d s\right| \leq & 2\|y(u)\|_{L^{\infty}(Q)} \\
& \times \int_{0}^{t}\left|\Phi_{\lambda, x}\right|_{2}\left|\Phi_{\lambda}\right|_{2} d s \\
\leq & \frac{\nu}{2} \int_{0}^{t}\left|\Phi_{\lambda, x}\right|_{2}^{2} d s+c_{1} \int_{0}^{t}\left|\Phi_{\lambda}\right|_{2}^{2} d s ;
\end{aligned}
$$

$$
\begin{aligned}
\left|-2 \int_{0}^{t}\left(\gamma \phi_{\lambda, x x x}, \Phi_{\lambda}\right)_{2} d s\right| & \left|2 \int_{0}^{t}\left(\gamma \phi_{\lambda, x x}, \Phi_{\lambda, x}\right)_{2} d s\right| \\
\leq & 2 \gamma \int_{0}^{t}\left|\Phi_{\lambda}\right|_{2}\left|\Phi_{\lambda, x}\right|_{2} d s \\
\leq & \frac{\nu}{2} \int_{0}^{t}\left|\Phi_{\lambda, x}\right|_{2}^{2} d s \\
& +c_{2} \int_{0}^{t}\left|\Phi_{\lambda}\right|_{2}^{2} d s \\
\left|2 \int_{0}^{t}\left(\epsilon(\lambda), \Phi_{\lambda}\right)_{2} d s\right| \leq & 2 \int_{0}^{t}|\epsilon(\lambda)|_{2}\left|\Phi_{\lambda}\right|_{2} d s \\
\leq & \int_{0}^{t}|\epsilon(\lambda)|_{2}^{2} d s+\int_{0}^{t}\left|\Phi_{\lambda}\right|_{2}^{2} d s
\end{aligned}
$$

where $c_{0}, c_{1}$, and $c_{2}$ are constants. We replace the right hand side of (62) by the right members of (61)-(65). And we apply 
the Gronwall inequality to the replaced inequality; then we arrive at

$$
\left|\Phi_{\lambda}(t)\right|_{2}^{2}+\int_{0}^{t}\left|\Phi_{\lambda, x}\right|_{2}^{2} d s \leq C\|\epsilon(\lambda)\|_{L^{2}\left(0, T ; L^{2}(\Omega)\right)}^{2},
$$

where $C$ is a constant. By virtue of (59) and (66) we deduce that

$$
\begin{array}{r}
\Phi_{\lambda} \longrightarrow 0 \quad \text { in } C\left([0, T] ; L^{2}(\Omega)\right) \cap L^{2}\left(0, T ; H_{0}^{1}(\Omega)\right) \\
\text { as } \lambda \longrightarrow 0 .
\end{array}
$$

As in (21)-(23), we have from (58), (66), and (67) that

$$
\Phi_{\lambda, t} \longrightarrow 0 \quad \text { strongly in } L^{2}\left(0, T ; H^{-1}(\Omega)\right) \text { as } \lambda \longrightarrow 0 \text {. }
$$

Therefore (67) and (68) mean

$$
\Phi_{\lambda} \longrightarrow 0 \text { strongly in } \mathscr{W}(0, T) \text { as } \lambda \longrightarrow 0 \text {. }
$$

Whence from Lemma 1

$$
z_{\lambda}(\cdot) \longrightarrow z(\cdot) \quad \text { strongly in } \mathcal{S}(0, T) \text { as } \lambda \longrightarrow 0 .
$$

This completes the proof.

Theorem 7 means that the cost $J(v)$ is Gâteaux differentiable at $u$ in the direction $v-u$ and the optimality condition (31) is rewritten by

$$
\begin{aligned}
\left(C y(u)-Y_{d}, C(D y(u)(v-u))\right)_{M}+(R u, v-u)_{u} \\
=\left\langle C^{*} \Lambda_{M}\left(C y(u)-Y_{d}\right), D y(u)(v-u)\right\rangle_{\mathcal{S}(0, T)^{\prime}, \mathcal{S}(0, T)} \\
\quad+(R u, v-u)_{u} \geq 0, \quad \forall v \in \mathcal{U}_{\mathrm{ad}},
\end{aligned}
$$

where $\Lambda_{M}$ is the canonical isomorphism $M$ onto $M^{\prime}$ and $Y_{d} \epsilon$ $M$ is a desired value.

3.3. Necessary Condition of Optimal Control. In this section we will characterize the optimal controls by giving necessary condition (71) for optimality for the following physically meaningful observations.

(1) We take $M=L^{2}(Q)$ and $C_{1} \in \mathscr{L}(\mathcal{S}(0, T), M)$ and observe

$$
z(v)=C_{1} y(v)=y(v ; \cdot) \in L^{2}(Q) .
$$

(2) We take $M=L^{2}(Q)$ and $C_{2} \in \mathscr{L}(\mathcal{S}(0, T), M)$ and observe

$$
z(v)=C_{2} y(v)=\left(I-\partial_{x}^{2}\right) y(v ; \cdot) \equiv m(v ; \cdot) \in L^{2}(Q)
$$

Since $y \in \mathcal{S}(0, T) \subset C\left([0, T] ; H_{0}^{2}(\Omega)\right)$ by Theorem 3 , the above observations are meaningful.

Due to Lions [10], we construct the necessary condition of optimal control via appropriate adjoint equation. In order to follow the idea we need to introduce and analyze the following adjoint equation for distributive observations:

$$
\begin{gathered}
-\mathscr{P}_{t}-v \mathscr{P}_{x x}-2 \omega p_{x}(u)-2(m(u) p(u))_{, x} \\
+\left(I-\partial_{x}^{2}\right)\left(2 y_{x}(u) p(u)\right)+m_{x}(u) p(u) \\
-\left(I-\partial_{x}^{2}\right)(y(u) p(u))_{, x}-\gamma p_{x x x}(u) \\
=C^{*} \Lambda_{M}\left(C y(u)-Y_{d}\right) \quad \text { in } Q, \\
p(u ; 0, t)=p(u ; 1, t)=p_{x}(u ; 0, t) \\
=p_{x}(u ; 1, t)=0, \quad t \in[0, T], \\
\mathscr{P}(x, T)=0 \text { in } \Omega,
\end{gathered}
$$

where $C=C_{1}$ or $C_{2}, \mathscr{P}=p(u)-p_{x x}(u)$, and $m(u)=$ $y(u)-y_{x x}(u)$. In order to show the well-posedness of (74), we introduce the solution Hilbert space $W\left(H_{0}^{2}(\Omega), L^{2}(\Omega)\right)$ defined by

$$
\begin{aligned}
& W\left(H_{0}^{2}(\Omega), L^{2}(\Omega)\right) \\
& \quad \triangleq\left\{\psi \mid \psi \in L^{2}\left(0, T ; H_{0}^{2}(\Omega)\right), \psi^{\prime} \in L^{2}\left(0, T ; L^{2}(\Omega)\right)\right\}
\end{aligned}
$$

equipped with the norm

$$
\|\psi\|_{W\left(H_{0}^{2}(\Omega), L^{2}(\Omega)\right)}=\left(\|\psi\|_{L^{2}\left(0, T ; H_{0}^{2}(\Omega)\right)}^{2}+\left\|\psi^{\prime}\right\|_{L^{2}\left(0, T ; L^{2}(\Omega)\right)}^{2}\right)^{1 / 2} .
$$

We remark that $W\left(H_{0}^{2}(\Omega), L^{2}(\Omega)\right)$ is continuously embedded in $C\left([0, T] ; H_{0}^{1}(\Omega)\right)$ (cf. Dautray and Lions [16, page 555$]$ ).

In the following proposition we show the well-posedness of (74).

Proposition 8. Assume that $C^{*} \Lambda_{M}\left(C y(u)-Y_{d}\right) \in L^{2}(0, T$; $H^{-2}(\Omega)$ ); then by reversing the direction of time $t \rightarrow T-t$, (74) admits a unique solution $p(u)$ satisfying

$$
\begin{aligned}
& \text { (i) } p(u) \in W\left(H_{0}^{2}(\Omega), L^{2}(\Omega)\right) \\
& \begin{aligned}
\text { (ii) }\left(p_{t}(u)-v p_{x x}(u)-y(u) p_{x}(u)+y_{x}(u) p(u), \Phi\right)_{2} \\
\quad-\left(2 \omega p_{x}(u)-m_{x}(u) p(u), \phi\right)_{2} \\
\quad+\left(2 m(u) p(u)+\gamma p_{x x}(u), \phi_{x}\right)_{2} \\
=\langle g, \phi\rangle_{-2,2}, \quad \forall \phi \in H_{0}^{2}(\Omega)
\end{aligned}
\end{aligned}
$$

in the sense of $\mathscr{D}^{\prime}(0, T)$,

(iii) $\mathscr{P}(x, 0)=0 \quad$ in $\Omega$,

where $g=C^{*} \Lambda_{M}\left(C y(u)-Y_{d}\right)$ and $\Phi=\phi-\phi_{x x}$.

The proof of Proposition 8 is given in the Appendix. 
Remark 9. As we will see, there are some merits in taking $W\left(H_{0}^{2}(\Omega), L^{2}(\Omega)\right)$ as the solution space for adjoint equations. For the observation (72), even though we can take the adjoint system in the space $\mathcal{S}(0, T)$ with additional boundary conditions, we can derive the same necessary optimality condition of optimal controls through the less regular solution $p(u) \epsilon$ $W\left(H_{0}^{2}(\Omega), L^{2}(\Omega)\right)$. Therefore, $W\left(H_{0}^{2}(\Omega), L^{2}(\Omega)\right)$ is preferred solution space of adjoint equation for the observation (72). And also, for the observation (73), we need to solve adjoint equation in $W\left(H_{0}^{2}(\Omega), L^{2}(\Omega)\right)$ because of the less regular data condition than that of the observation (72).

3.3.1. Case of the Observation (72). In this subsection we consider the cost functional expressed by

$$
\begin{array}{r}
J(v)=\int_{Q}\left|y(v ; x, t)-Y_{d}(x, t)\right|^{2} d x d t+(R v, v)_{\mathscr{U}}, \\
\forall v \in \mathcal{U}_{\mathrm{ad}} \subset \mathcal{U},
\end{array}
$$

where $Y_{d} \in L^{2}(Q)$ is a desired value. Let $u$ be the optimal control subject to (27) and (78). Then the optimality condition (71) is represented by

$$
\int_{Q}\left(y(u ; x, t)-Y_{d}(x, t)\right) z(x, t) d x d t+(R u, v-u)_{u} \geq 0
$$

$\forall v \in \mathcal{U}_{\mathrm{ad}}$

where $z$ is the solution of (47).

Now we will formulate the following adjoint system to describe the optimality condition:

$$
\begin{gathered}
-\mathscr{P}_{t}-v \mathscr{P}_{x x}-2 \omega p_{x}(u)-2(m(u) p(u))_{, x} \\
+\left(I-\partial_{x}^{2}\right)\left(2 y_{x}(u) p(u)\right) \\
+m_{x}(u) p(u)-\left(I-\partial_{x}^{2}\right)(y(u) p(u))_{, x} \\
-\gamma p_{x x x}(u)=y(u)-Y_{d} \quad \text { in } Q, \\
p(u ; 0, t)=p(u ; 1, t)=p_{x}(u ; 0, t) \\
=p_{x}(u ; 1, t)=0, \quad t \in[0, T], \\
\mathscr{P}(x, T)=0 \quad \text { in } \Omega,
\end{gathered}
$$

where $\mathscr{P}=p(u)-p_{x x}(u)$ and $m(u)=y(u)-y_{x x}(u)$.

Remark 10. Taking into account the observation conditions $y(u)-Y_{d} \in L^{2}(Q)=L^{2}\left(0, T ; L^{2}(\Omega)\right) \subset L^{2}\left(0, T ; H^{-2}(\Omega)\right)$, we can assert that (80), reversing the direction of time $t \rightarrow$ $T-t$, admits a unique solution $p(u) \in W\left(H_{0}^{2}(\Omega), L^{2}(\Omega)\right)$ by Proposition 8.
Now we proceed the calculations. We multiply both sides of the weak form of $(80)$ by $z(t)$ and integrate it over $[0, T]$. Then we have

$$
\begin{aligned}
& \int_{0}^{T}\left\langle-\mathscr{P}_{t}-v \mathscr{P}_{x x}-2 \omega p_{x}(u)-2(m(u) p(u))_{, x}\right. \\
& \left.\quad+\left(I-\partial_{x}^{2}\right)\left(2 y_{x}(u) p(u)\right), z\right\rangle_{-2,2} d t \\
& +\int_{0}^{T}\left\langle m_{x}(u) p(u)-\left(I-\partial_{x}^{2}\right)(y(u) p(u))_{, x}\right. \\
& \left.\quad-\gamma p_{x x x}(u), z\right\rangle_{-1,1} d t \\
& =\int_{0}^{T}\left(y(u)-Y_{d}, z\right)_{2} d t,
\end{aligned}
$$

where $\mathscr{P}=p(u)-p_{x x}(u)$. By $(47)$ for $z$, we can verify by integration by parts that the left hand side of (81) yields

$$
\begin{aligned}
& \int_{0}^{T}\left(p(u), \mathscr{X}_{t}-v \mathscr{E}_{x x}+2 \omega z_{x}+2 z_{x} m(u)\right. \\
& \left.\quad+2 y_{x}(u) \mathscr{Z}+m_{x}(u) z+y(u) \mathscr{Z}_{x}+\gamma z_{x x x}\right)_{2} d t \\
& \quad=\int_{0}^{T}(p(u), B(v-u))_{2} d t,
\end{aligned}
$$

where $\mathscr{Z}=z-z_{x x}$. Therefore, by (81) and (82) we can deduce that the optimality condition (79) is equivalent to

$$
\int_{0}^{T}(p(u), B(v-u))_{2} d t+(R u, v-u)_{\mathscr{U}} \geq 0, \quad \forall v \in \mathscr{U}_{\mathrm{ad}}
$$

Hence, we give the following theorem.

Theorem 11. The optimal control $u$ for $(78)$ is characterized by the following system of equations and inequality:

$$
\begin{gathered}
m_{t}(u)-v m_{x x}(u)+2 \omega y_{x}(u)+2 y_{x}(u) m(u) \\
+y(u) m_{x}(u)+\gamma y_{x x x}(u) \\
=f+B u \quad \text { in } Q \\
y(u ; 0, t)=y(u ; 1, t)=y_{x}(u ; 0, t) \\
=y_{x}(u ; 1, t)=y_{x x}(u ; 0, t) \\
=y_{x x}(u ; 1, t)=0, \quad t \in[0, T] \\
m(u ; x, 0)=m_{0}=y_{0}-y_{0, x x}, \quad \text { in } \Omega,
\end{gathered}
$$




$$
\begin{gathered}
-\mathscr{P}_{t}-v \mathscr{P}_{x x}-2 \omega p_{x}(u)-2(m(u) p(u))_{, x} \\
+\left(I-\partial_{x}^{2}\right)\left(2 y_{x}(u) p(u)\right)+m_{x}(u) p(u) \\
\quad-\left(I-\partial_{x}^{2}\right)(y(u) p(u))_{, x}-\gamma p_{x x x}(u) \\
=y(u)-Y_{d} \quad \text { in } Q, \\
p(u ; 0, t)=p(u ; 1, t)=p_{x}(u ; 0, t) \\
=p_{x}(u ; 1, t)=0, \quad t \in[0, T], \\
\mathscr{P}(x, T)=0 \quad \text { in } \Omega, \\
\int_{0}^{T}(p(u), B(v-u))_{2} d t+(R u, v-u)_{u} \geq 0,
\end{gathered}
$$$$
\forall v \in \mathcal{U}_{\text {ad }},
$$

where $m(u)=y(u)-y_{x x}(u)$ and $\mathscr{P}=p(u)-p_{x x}(u)$.

3.3.2. Case of the Observation (73). We consider the following momentum's distributive cost functional expressed by

$$
J(v)=\int_{Q}\left|m(v)-Y_{d}\right|^{2} d x d t+(R v, v)_{\mathcal{U}}, \quad \forall v \in \mathcal{U}_{\mathrm{ad}},
$$

where $m(v)=y(v)-y_{x x}(v)$ and $Y_{d} \in L^{2}(Q)$. Let $u$ be the optimal control subject to (27) and (87). Then the optimality condition (71) is rewritten as

$$
\int_{0}^{T}\left(m(u)-Y_{d}, \mathscr{Z}\right)_{2} d x d t+(R u, v-u)_{\mathscr{U}} \geq 0, \quad \forall v \in \mathscr{U}_{\mathrm{ad}},
$$

where $\mathscr{Z}=z-z_{x x}$ and $z$ is the solution of (47). As before we formulate the following adjoint system to describe the optimality condition:

$$
\begin{gathered}
-\mathscr{P}_{t}-v \mathscr{P}_{x x}-2 \omega p_{x}(u)-2(m(u) p(u))_{, x} \\
+\left(I-\partial_{x}^{2}\right)\left(2 y_{x}(u) p(u)\right)+m_{x}(u) p(u) \\
-\left(I-\partial_{x}^{2}\right)(y(u) p(u))_{, x}-\gamma p_{x x x}(u) \\
=\left(I-\partial_{x}^{2}\right)\left(m(u)-Y_{d}\right) \quad \text { in } Q, \\
p(u ; 0, t)=p(u ; 1, t)=p_{x}(u ; 0, t) \\
=p_{x}(u ; 1, t)=0, \quad t \in[0, T], \\
\mathscr{P}(x, T)=0 \quad \text { in } \Omega,
\end{gathered}
$$

where $\mathscr{P}=p(u)-p_{x x}(u)$ and $m(u)=y(u)-y_{x x}(u)$.

Remark 12. Since the observation conditions $m(u)-Y_{d} \in$ $L^{2}(Q)=L^{2}\left(0, T ; L^{2}(\Omega)\right)$, we know that $\left(I-\partial_{x}^{2}\right)\left(m(u)-Y_{d}\right) \epsilon$ $L^{2}\left(0, T ; H^{-2}(\Omega)\right)$. Hence by reversing the direction of time $t \rightarrow T-t$ and applying Proposition 8, we deduce that (89) admits a unique solution $p(u) \in W\left(H_{0}^{2}(\Omega), L^{2}(\Omega)\right)$.
As we did before, we multiply both sides of the weak form of (89) by $z(t)$ and integrate it over $[0, T]$. Then we have

$$
\begin{gathered}
\int_{0}^{T}\left\langle-\mathscr{P}_{t}-v \mathscr{P}_{x x}-2 \omega p_{x}(u)-2(m(u) p(u))_{, x}\right. \\
\left.\quad+\left(I-\partial_{x}^{2}\right)\left(2 y_{x}(u) p(u)\right), z\right\rangle_{-2,2} d t \\
\quad+\int_{0}^{T}\left\langle m_{x}(u) p(u)-\left(I-\partial_{x}^{2}\right)(y(u) p(u))_{, x}\right. \\
\left.\quad-\gamma p_{x x x}(u), z\right\rangle_{-1,1} d t \\
=\int_{0}^{T}\left\langle\left(I-\partial_{x}^{2}\right)\left(m(u)-Y_{d}\right), z\right\rangle_{-2,2} d t \\
=\int_{0}^{T}\left(m(u)-Y_{d}, \mathscr{Z}\right)_{2} d t
\end{gathered}
$$

where $\mathscr{P}=p(u)-p_{x x}(u), m(u)=y(u)-y_{x x}(u)$, and $\mathscr{Z}=$ $z-z_{x x}$. By (47) for $z$ the integration by parts of the left hand side of (90) yields

$$
\begin{aligned}
& \int_{0}^{T}\left(p(u), \mathscr{Z}_{t}-v \mathscr{Z}_{x x}+2 \omega z_{x}+2 z_{x} m(u)+2 y_{x}(u) \mathscr{Z}\right. \\
& \left.\quad+m_{x}(u) z+y(u) \mathscr{Z}_{x}+\gamma z_{x x x}\right)_{2} d t \\
& =\int_{0}^{T}(p(u), B(v-u))_{2} d t,
\end{aligned}
$$

where $\mathscr{Z}=z-z_{x x}$. Therefore, combining (90) and (91), we can deduce that the optimality condition (88) is equivalent to

$$
\int_{0}^{T}(p(u), B(v-u))_{2} d t+(R u, v-u)_{u} \geq 0, \quad \forall v \in \mathcal{U}_{\mathrm{ad}}
$$

Hence, we give the following theorem.

Theorem 13. The optimal control $u$ for (87) is characterized by the following system of equations and inequality:

$$
\begin{gathered}
m_{t}(u)-v m_{x x}(u)+2 \omega y_{x}(u)+2 y_{x}(u) m(u) \\
+y(u) m_{x}(u)+\gamma y_{x x x}(u)=f+B u \quad \text { in } Q, \\
y(u ; 0, t)=y(u ; 1, t)=y_{x}(u ; 0, t) \\
=y_{x}(u ; 1, t)=y_{x x}(u ; 0, t) \\
=y_{x x}(u ; 1, t)=0, \quad t \in[0, T], \\
m(u ; x, 0)=m_{0}=y_{0}-y_{0, x x} \quad \text { in } \Omega,
\end{gathered}
$$




$$
\begin{aligned}
&-\mathscr{P}_{t}--\mathscr{P}_{x x}-2 \omega p_{x}(u)-2(m(u) p(u))_{, x} \\
&+\left(I-\partial_{x}^{2}\right)\left(2 y_{x}(u) p(u)\right)+m_{x}(u) p(u) \\
&-\left(I-\partial_{x}^{2}\right)(y(u) p(u))_{, x}-\gamma p_{x x x}(u) \\
&=\left(I-\partial_{x}^{2}\right)\left(m(u)-Y_{d}\right) \quad \text { in } Q, \\
& p(u ; 0, t)=p(u ; 1, t)=p_{x}(u ; 0, t) \\
&=p_{x}(u ; 1, t)=0, \quad t \in[0, T], \\
& \mathscr{P}(x, T)=0 \quad \text { in } \Omega, \\
& \int_{0}^{T}(p(u), B(v-u))_{2} d t+(R u, v-u)_{\mathcal{U}} \geq 0, \quad \forall v \in \mathcal{U}_{\mathrm{ad}},
\end{aligned}
$$

where $m(u)=y(u)-y_{x x}(u)$ and $\mathscr{P}=p(u)-p_{x x}(u)$.

3.4. Local Uniqueness of an Optimal Control. We note that the uniqueness of an optimal control in nonlinear problem is not assured. However, referring to the result in [15], we can show the local uniqueness of the optimal control for our problem. In order to show the uniqueness of optimal control by using strict convexity of quadratic cost (cf. [18]) we consider the following proposition.

Proposition 14. The map $v \rightarrow y(v)$ of $\mathcal{U}$ into $\mathcal{S}(0, T)$ is second order Gâteaux differentiable at $v=u$ and such the second order Gâteaux derivative of $y(v)$ at $v=u$ in the direction $v-u \in U$, say $g=D^{2} y(u)(v-u, v-u)$, is a unique solution of the following problem:

$$
\begin{aligned}
& \mathscr{G}_{t}-v \mathscr{G}_{x x}+2 \omega g_{x}+y(u) \mathscr{G}_{x}+2 y_{x}(u) \mathscr{G}+2 g_{x} m \\
& +g m_{x}+4 z_{x} \mathscr{E}+2 z \mathscr{E}_{x}+\gamma g_{x x x}=0 \quad \text { in } \mathrm{Q}, \\
& g(0, t)=g(1, t)=g_{x}(0, t)=g_{x}(1, t)=g_{x x}(0, t) \\
& \quad=g_{x x}(1, t)=0, \quad t \in[0, T], \\
& \mathscr{G}(x, 0)=0 \quad \text { in } \Omega,
\end{aligned}
$$

where $\mathscr{G}=g-g_{x x}, \mathscr{Z}=z-z_{x x}$, and $z$ is the solution of (47).

Proof. The proof is similar to that of Theorem 7.

Lemma 15. Let $g$ be the solution of (96). Then we can show that

$$
\|g\|_{\mathcal{S}(0, T)} \leq C\|v-u\|_{\mathscr{U}}^{2},
$$

where $C>0$ is a constant.

Proof. Let $z$ be the solution of (47). Then, using the same arguments as in (5), we can deduce that

$$
\begin{aligned}
\|\mathscr{E}\|_{\mathscr{W}(0, T)} & \leq c_{0}\|B(v-u)\|_{L^{2}\left(0, T ; L^{2}(\Omega)\right)} \\
& \leq c_{0}\|B\|_{\mathscr{L}\left(\mathcal{U} ; L^{2}\left(0, T ; L^{2}(\Omega)\right)\right)}\|v-u\|_{\mathscr{U}} \\
& \leq c_{1}\|v-u\|_{\mathscr{U}},
\end{aligned}
$$

where $\mathscr{Z}=z-z_{x x}$ and $c_{0}, c_{1}$ are constants which does not depend on $g$. And also for the solution $g$ of (96), we can show that

$$
\begin{aligned}
\|g\|_{\mathcal{S}(0, T)} & \leq c_{2}\left\|-4 z_{x} \mathscr{Z}-2 z \mathscr{E}_{x}\right\|_{L^{2}\left(0, T L^{2}(\Omega)\right)} \\
& \leq c_{3}\left(\left\|z_{x}\right\|_{L^{\infty}(\mathrm{Q})}+\|z\|_{L^{\infty}(Q)}\right)\|\mathscr{E}\|_{L^{2}\left(0, T ; H_{0}^{1}(\Omega)\right)} \\
& \leq c_{4}\|z\|_{\mathcal{S}(0, T)}\|\mathscr{E}\|_{\mathscr{W}(0, T)} \\
& \leq c_{5}\|\mathscr{E}\|_{\mathscr{W}(0, T)}^{2},
\end{aligned}
$$

where $c_{2}, \ldots, c_{5}$ are constants. Combining (98) with (99), we have (97).

We prove the local uniqueness of the optimal control.

Theorem 16. When $T$ is small enough, then there is a unique optimal control for the problem (29) for observations (72) and (73).

Proof. We prove the case (73). Then the same result will be followed for the case (72).

We show the local uniqueness by proving the strict convexity of the map $v \in \mathscr{U}_{\mathrm{ad}} \rightarrow J(v)$. Therefore as in [18], we need to show for all $u, v \in \mathscr{U}_{\text {ad }}(u \neq v)$

$$
D^{2} J(u+\xi(v-u))(v-u, v-u)>0 \quad(0<\xi<1) .
$$

For simplicity, we denote $y(u+\xi(v-u)), z(u+\xi(v-u))$, and $g(u+\xi(v-u))$ by $y(\xi), z(\xi)$, and $g(\xi)$, respectively.

We calculate

$$
\begin{aligned}
& D J(u+\xi(v-u))(v-u) \\
&= \lim _{l \rightarrow 0} \frac{J(u+(\xi+l)(v-u))-J(u+\xi(v-u))}{l} \\
&=2 \int_{0}^{T}\left(m(\xi)-Y_{d}, \mathscr{Z}(\xi)\right)_{2} d s \\
& \quad+2(R(u+\xi(v-u)), v-u)_{\mathcal{U}},
\end{aligned}
$$

where $m(\xi)=y(\xi)-y_{x x}(\xi)$ and $\mathscr{Z}(\xi)=z(\xi)-z_{x x}(\xi)$. From (101) we obtain the second order Gâteaux derivative of $J$ as follows:

$$
\begin{aligned}
& D^{2} J(u+\xi(v-u))(v-u, v-u) \\
& =\lim _{k \rightarrow 0}((D J(u+(\xi+k)(v-u))(v-u) \\
& \left.\quad-D J(u+\xi(v-u))(v-u)) \times k^{-1}\right) \\
& =2 \int_{0}^{T}\left(m(\xi)-Y_{d}, \mathscr{G}(\xi)\right)_{2} d s+2 \int_{0}^{T}|\mathscr{Z}(\xi)|_{2}^{2} d s \\
& \quad+2(R(v-u), v-u)_{\mathcal{U}} \\
& =2 \int_{0}^{T}\left\langle\left(I-\partial_{x}^{2}\right)\left(m(\xi)-Y_{d}\right), g(\xi)\right\rangle_{-2,2} d s \\
& +2 \int_{0}^{T}|\mathscr{Z}(\xi)|_{2}^{2} d s+2(R(v-u), v-u)_{\mathscr{U}},
\end{aligned}
$$

where $\mathscr{G}(\xi)=g(\xi)-g_{x x}(\xi)$. 
By Lemma 15 and (102) we deduce that

$$
\begin{aligned}
D^{2} J( & +\xi(v-u))(v-u, v-u) \\
\geq & -2\|g(\xi)\|_{L^{\infty}\left(0, T ; H_{0}^{2}(\Omega)\right)} \\
& \times \int_{0}^{T}\left\|\left(I-\partial_{x}^{2}\right)\left(m(\xi)-Y_{d}\right)\right\|_{H^{-2}(\Omega)} d s \\
& +2 \int_{0}^{T}|\mathscr{Z}(\xi)|_{2}^{2} d s+2 d\|v-u\|_{\mathscr{U}}^{2} \\
\geq & -2 c_{0} \sqrt{T}\|g(\xi)\|_{\delta(0, T)}\left\|m(\xi)-Y_{d}\right\|_{L^{2}\left(0, T ; L^{2}(\Omega)\right)} \\
& +2 \int_{0}^{T}|\mathscr{Z}(\xi)|_{2}^{2} d s+2 d\|v-u\|_{\mathscr{U}}^{2} \\
\geq & 2\left(d-c_{1} \sqrt{T}\left\|_{m}(\xi)-Y_{d}\right\|_{L^{2}\left(0, T ; L^{2}(\Omega)\right)}\right)\|v-u\|_{\mathscr{U}}^{2} \\
& +2 \int_{0}^{T}|\mathscr{Z}(\xi)|_{2}^{2} d s,
\end{aligned}
$$

where $c_{0}$ and $c_{1}$ are constants. Here we can take $T>0$ small enough so that the right hand side of (103) is strictly greater than 0 . Therefore we obtain the strict convexity of the quadratic cost $J(v), v \in \mathcal{U}_{\text {ad }}$, which prove this theorem.

Remark 17. If we assume $d$ is large enough then we can obtain the strict convexity of the quadratic cost (29) in global sense. Therefore we can obtain the desired result of Theorem 16 in global sense for the cost (29).

\section{Conclusions}

In conclusion, in this paper we considered the optimal distributed control for the viscous Dullin-Gottwald-Holm equation due to Lions [10]. In order to apply the variational approach due to Lions [10] to our problem, we proposed the quadratic cost functional as studied in Lions [10] which is to be minimized within an admissible set of control variables. We showed the existence of optimal controls which minimizes the quadratic cost functional. Then, we established the necessary conditions of optimality of the optimal control for some physically meaningful observation cases employing the associate adjoint systems. For this we successfully proved the Gâteaux differentiability of the nonlinear solution mapping which is used to define the associate adjoint systems. Moreover, by proving strict convexity of the quadratic cost with respect to the control variable, we discussed the local uniqueness of optimal control.

\section{Appendix}

Proof of Proposition 8. For simplicity we omit $u$ in (74) and put $C^{*} \Lambda_{M}\left(C y(u)-Y_{d}\right)=f$. By reversing time $t \rightarrow T-t$, (74) is transformed as

$$
\begin{aligned}
\mathscr{P}_{t}- & \nu \mathscr{P}_{x x}-2 \omega p_{x}-2(m p)_{, x}+\left(I-\partial_{x}^{2}\right)\left(2 y_{x} p\right) \\
& +m_{x} p-\left(I-\partial_{x}^{2}\right)(y p)_{, x}-\gamma p_{x x x}=f \text { in } Q,
\end{aligned}
$$

$$
\begin{aligned}
p(0, t) & =p(1, t)=p_{x}(0, t) \\
& =p_{x}(1, t)=0, \quad t \in[0, T], \\
\mathscr{P}(x, 0) & =0 \quad \text { in } \Omega .
\end{aligned}
$$

We apply the Galerkin procedure as in Dautray and Lions [16]. Let $\left\{w_{n}\right\}_{n=1}^{\infty}$ be a basis of $H_{0}^{2}(\Omega)$. For each $n \in N$ we define an approximate solution of (A.1) by $p_{n}(t)=\sum_{j=1}^{n} g_{j n}(t) w_{j}$ which satisfies

$$
\begin{aligned}
&\left\langle\mathscr{P}_{n, t},\right.\left.w_{j}\right\rangle_{-2,2}+v\left\langle\mathscr{P}_{n, x}, w_{j, x}\right\rangle_{-1,1} \\
&-\left(2 \omega p_{n, x}, w_{j}\right)_{2}+\left(2 m p_{n}, w_{j, x}\right)_{2} \\
&+\left(2 y_{x} p_{n},\left(I-\partial_{x}^{2}\right) w_{j}\right)_{2}+\left(m_{x} p_{n}, w_{j}\right)_{2} \\
&-\left(y_{x} p_{n}+y p_{n, x},\left(I-\partial_{x}^{2}\right) w_{j}\right)_{2} \\
&-\left(\gamma p_{n, x x x}, w_{j}\right)_{2}=\left\langle f, w_{j}\right\rangle_{-2,2}, \quad 1 \leq j \leq n, \\
& \mathscr{P}_{n}(x, 0)=0 \quad \text { in } \Omega,
\end{aligned}
$$

where $\mathscr{P}_{n}=p_{n}-p_{n, x x}$. We multiply both sides of (A.2) by $g_{\text {jn }}(t)$ and sum over $j$ to have

$$
\begin{aligned}
\left\langle\mathscr{P}_{n, t}\right. & \left., p_{n}\right\rangle_{-2,2}+\nu\left\langle\mathscr{P}_{n, x}, p_{n, x}\right\rangle_{-1,1}+\left(2 m p_{n}, p_{n, x}\right)_{2} \\
& +\left(2 y_{x} p_{n}, p_{n}-p_{n, x x}\right)_{2}+\left(m_{x} p_{n}, p_{n}\right)_{2} \\
& -\left(y_{x} p_{n}+y p_{n, x}, p_{n}-p_{n, x x}\right)_{2} \\
& -\left(\gamma p_{n, x x x}, p_{n}\right)_{2}=\left\langle f, p_{n}\right\rangle_{-2,2} .
\end{aligned}
$$

We note here that

$$
\left(2 \omega p_{n, x}, p_{n}\right)_{2}=\left(\gamma p_{n, x x x}, p_{n}\right)_{2}=0 \text { a.e. in }[0, T] \text {. }
$$

Hence we can rewrite (A.3) as follows:

$$
\begin{aligned}
\frac{1}{2} \frac{d}{d t}\left(\left|p_{n}\right|_{2}^{2}+\left|p_{n, x}\right|_{2}^{2}\right)+v\left(\left|p_{n, x}\right|_{2}^{2}+\left|p_{n, x x}\right|_{2}^{2}\right) \\
=-\left(2 m p_{n}, p_{n, x}\right)_{2}-\left(2 y_{x} p_{n}, p_{n}-p_{n, x x}\right)_{2}-\left(m_{x} p_{n}, p_{n}\right)_{2} \\
\quad+\left(y_{x} p_{n}+y p_{n, x}, p_{n}-p_{n, x x}\right)_{2}+\left\langle f, p_{n}\right\rangle .
\end{aligned}
$$

By employing Schwartz's, Young's inequality and Sobolev imbedding theorem, we can estimate the right hand side of (A.5) as follows:

$$
\begin{aligned}
& \left|\left(2 m p_{n}, p_{n, x}\right)_{2}\right| \leq 2\|m\|_{L^{\infty}(\Omega)}\left|p_{n}\right|_{2}\left|p_{n, x}\right|_{2} \\
& \quad \leq c_{0}\left|m_{x}\right|_{2}\left(\left|p_{n}\right|_{2}^{2}+\left|p_{n, x}\right|_{2}^{2}\right) ; \\
& \left|\left(2 y_{x} p_{n}, p_{n}\right)_{2}\right| \leq c_{1}\left|p_{n}\right|_{2}^{2} ; \\
& \left|\left(2 y_{x} p_{n}, p_{n, x x}\right)_{2}\right| \leq c_{2}\left|p_{n}\right|_{2}\left|p_{n, x x}\right|_{2} \leq c_{3}(\epsilon)\left|p_{n}\right|_{2}^{2}+\epsilon\left|p_{n, x x}\right|_{2}^{2} ;
\end{aligned}
$$




$$
\begin{aligned}
& \left|\left(m_{x} p_{n}, p_{n}\right)_{2}\right| \leq\left\|p_{n}\right\|_{L^{\infty}(\Omega)}\left|m_{x}\right|_{2}\left|p_{n}\right|_{2} \leq c_{4}\left|p_{n, x}\right|_{2}\left|m_{x}\right|_{2}\left|p_{n}\right|_{2} \\
& \leq c_{4}\left|m_{x}\right|_{2}\left(\left|p_{n}\right|_{2}^{2}+\left|p_{n, x}\right|_{2}^{2}\right) \text {; } \\
& \left|\left(y_{x} p_{n}, p_{n}\right)_{2}\right| \leq c_{5}\left|p_{n}\right|_{2}^{2} \text {; } \\
& \left|\left(y_{x} p_{n}, p_{n, x x}\right)_{2}\right| \leq c_{6}\left|p_{n}\right|_{2}\left|p_{n, x x}\right|_{2} \leq c_{7}(\epsilon)\left|p_{n}\right|_{2}^{2}+\epsilon\left|p_{n, x x}\right|_{2}^{2} ; \\
& \left|\left(y p_{n, x}, p_{n}\right)_{2}\right| \leq c_{8}\left|p_{n, x}\right|_{2}\left|p_{n}\right|_{2} \leq c_{8}\left(\left|p_{n, x}\right|_{2}^{2}+\left|p_{n}\right|_{2}^{2}\right) ; \\
& \left|\left(y p_{n, x}, p_{n, x x}\right)_{2}\right| \leq c_{9}\left|p_{n, x}\right|_{2}\left|p_{n, x x}\right|_{2} \leq c_{10}(\epsilon)\left|p_{n, x}\right|_{2}^{2}+\epsilon\left|p_{n, x x}\right|_{2}^{2} ; \\
& \left|\left\langle f, p_{n}\right\rangle\right| \leq\|f\|_{H^{-2}(\Omega)}\left\|p_{n}\right\|_{H_{0}^{2}(\Omega)} \\
& \leq c_{11}(\epsilon)\|f\|_{H^{-2}(\Omega)}^{2}+\epsilon\left|p_{n, x x}\right|_{2}^{2},
\end{aligned}
$$

where $c_{0}, \ldots, c_{11}$ are constants. We put $\epsilon=\nu / 8$ and replace the right hand side of (A.5) by those right members of (A.6) to obtain

$$
\begin{aligned}
& \frac{d}{d t}\left(\left|p_{n}\right|_{2}^{2}+\left|p_{n, x}\right|_{2}^{2}\right)+\left(\left|p_{n, x}\right|_{2}^{2}+\left|p_{n, x x}\right|_{2}^{2}\right) \\
& \quad \leq c_{12}\left(1+\left|m_{x}\right|_{2}\right)\left(\left|p_{n}\right|_{2}^{2}+\left|p_{n, x}\right|_{2}^{2}\right)+c_{13}\|f\|_{H^{-2}(\Omega)}^{2},
\end{aligned}
$$

where $c_{12}$ and $c_{13}$ are constants. Integrating (A.7) over $[0, t]$ and applying the Gronwall's inequality to it, we have

$$
\begin{aligned}
& \left|p_{n}(t)\right|_{2}^{2}+\left|p_{n, x}(t)\right|_{2}^{2}+\int_{0}^{t}\left(\left|p_{n, x}\right|_{2}^{2}+\left|p_{n, x x}\right|_{2}^{2}\right) d s \\
& \quad \leq c_{13} \exp \left(c_{12} \int_{0}^{T}\left(1+\left|m_{x}\right|_{2}\right) d t\right)\|f\|_{L^{2}\left(0, T ; H^{-2}(\Omega)\right)}^{2} .
\end{aligned}
$$

Thus we know that

$$
\begin{aligned}
& p_{n} \in \text { a bounded subset of } \\
& \qquad L^{\infty}\left(0, T ; H_{0}^{1}(\Omega)\right) \cap L^{2}\left(0, T ; H_{0}^{2}(\Omega)\right) .
\end{aligned}
$$

Hence by replacing $p$ by $p_{n}$ in (A.1) and dividing $\left(I-\partial_{x}^{2}\right)$, we obtain the following equality:

$$
\begin{aligned}
p_{n, t}= & v p_{n, x x}+2 \omega\left(I-\partial_{x}^{2}\right)^{-1} p_{n, x}+2\left(I-\partial_{x}^{2}\right)^{-1}\left(m p_{n}\right)_{, x} \\
& -2 y_{x} p_{n}-\left(I-\partial_{x}^{2}\right)^{-1}\left(m_{x} p_{n}\right) \\
& +\left(y p_{n}\right)_{, x}+\gamma\left(I-\partial_{x}^{2}\right)^{-1} p_{n, x x x} \\
& +\left(I-\partial_{x}^{2}\right)^{-1} f \in L^{2}\left(0, T ; L^{2}(\Omega)\right)
\end{aligned}
$$

which implies via (A.9) that

$$
p_{n, t} \in \text { a bounded subset of } L^{2}\left(0, T ; L^{2}(\Omega)\right) \text {. }
$$

Therefore we have the boundedness of $\left\{p_{n}\right\}$ in $W\left(H_{0}^{2}(\Omega)\right.$, $L^{2}(\Omega)$ ). Hence by standard manipulations of Dautray and Lions [16], we can know that there exists a unique limit $p$ of $\left\{p_{n}\right\}$ in $W\left(H_{0}^{2}(\Omega), L^{2}(\Omega)\right)$ which is the unique solution of (A.1). This proves the well-posedness of (74).

\section{Conflict of Interests}

The author declares that there is no conflict of interests regarding the publication of this paper.

\section{Acknowledgment}

This paper was supported by the Daegu University Research Grant 2010.

\section{References}

[1] H. R. Dullin, G. A. Gottwald, and D. D. Holm, "An integrable shallow water equation with linear and nonlinear dispersion," Physical Review Letters, vol. 87, pp. 194501-194504, 2001.

[2] R. Camassa and D. D. Holm, "An integrable shallow water equation with peaked solitons," Physical Review Letters, vol. 71, no. 11, pp. 1661-1664, 1993.

[3] C. Shen and A. Gao, "Optimal control of the viscous weakly dispersive Degasperis-Procesi equation," Nonlinear Analysis: Theory, Methods and Applications, vol. 72, no. 2, pp. 933-945, 2009.

[4] X. Ai, L. Tian, C. Shen, and G. Guilong, "On the low regularity solutions for the generalized Dullin-Gottwald-Holm equation," International Journal of NonLinear Science, vol. 7, pp. 113-118, 2009.

[5] O. G. Mustafa, "Existence and uniqueness of low regularity solutions for the dullin-gottwald-holm equation," Communications in Mathematical Physics, vol. 265, no. 1, pp. 189-200, 2006.

[6] L. Tian, G. Gui, and Y. Liu, "On the well-posedness problem and the scattering problem for the Dullin-Gottwald-Holm equation," Communications in Mathematical Physics, vol. 257, no. 3, pp. 667-701, 2005.

[7] C. Shen, L. Tian, and A. Gao, "Optimal control of the viscous Dullin-Gottwalld-Holm equation," Nonlinear Analysis: Real World Applications, vol. 11, no. 1, pp. 480-491, 2010.

[8] D. D. Holm and M. F. Staley, "Wave structure and nonlinear balances in a family of $1+1$ evolutionary PDEs," SIAM Journal on Applied Dynamical Systems, vol. 2, no. 1, pp. 323-380, 2003.

[9] B. Sun, "Maximum principle for optimal distributed control of the viscous DullinGottwaldHolm equation," Nonlinear Analysis: Real World Applications, vol. 13, no. 1, pp. 325-332, 2012.

[10] J. L. Lions, Optimal Control of Systems Governed by Partial Differential Equations, Springer, Berlin, Germany, 1971.

[11] J.-S. Hwang and S.-I. Nakagiri, "Optimal control problems for Kirchhoff type equation with a damping term," Nonlinear Analysis: Theory, Methods and Applications, vol. 72, no. 3-4, pp. 16211631, 2010.

[12] J.-S. Hwang and S.-I. Nakagiri, "Optimal control problems for the equation of motion of membrane with strong viscosity," Journal of Mathematical Analysis and Applications, vol. 321, no. 1, pp. 327-342, 2006.

[13] J.-S. Hwang, "Optimal control problems for an extensible beam equation," Journal of Mathematical Analysis and Applications, vol. 353, no. 1, pp. 436-448, 2009.

[14] J. Hwang, "Parameter identification problems for an extensible beam equation," Journal of Mathematical Analysis and Applications, vol. 359, pp. 682-695, 2009.

[15] S.-U. Ryu, "Optimal control problems governed by some semilinear parabolic equations," Nonlinear Analysis, Theory, Methods and Applications, vol. 56, no. 2, pp. 241-252, 2004. 
[16] R. Dautray and J. L. Lions, Mathematical Analysis and Numerical Methods for Science and Technology, vol. 5 of Evolution Problems I, Springer, 1992.

[17] R. Temam, Navier Stokes Equation, North-Holland, 1984.

[18] E. Zeidler, NonLinear Functional Analysis and Its Applications, $I I / B$, NonLinear Monotone Operator, Springer, Berlin, Germany, 1990. 


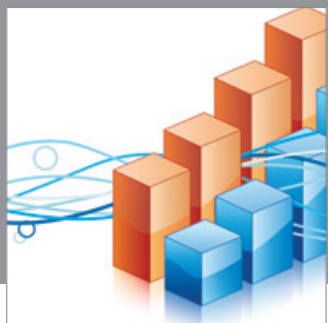

Advances in

Operations Research

mansans

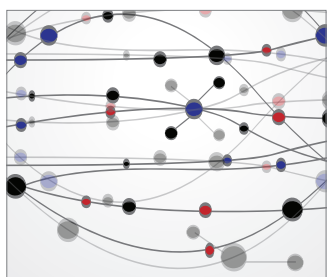

The Scientific World Journal
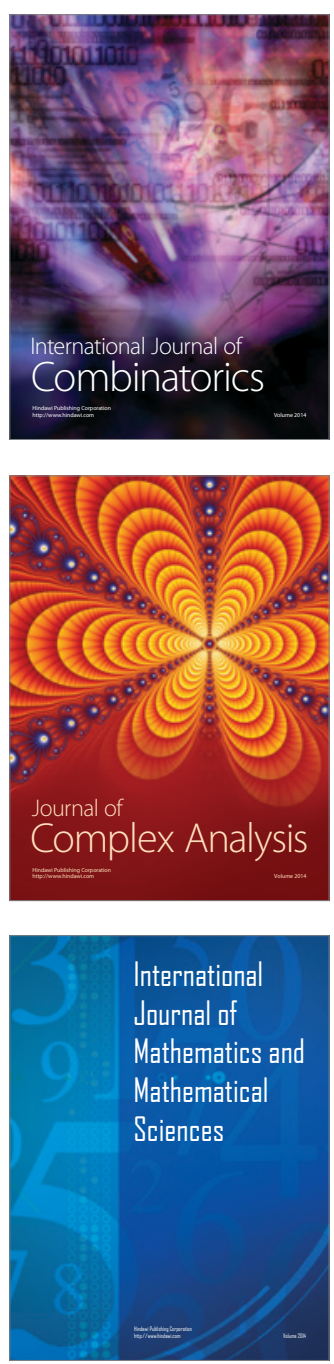
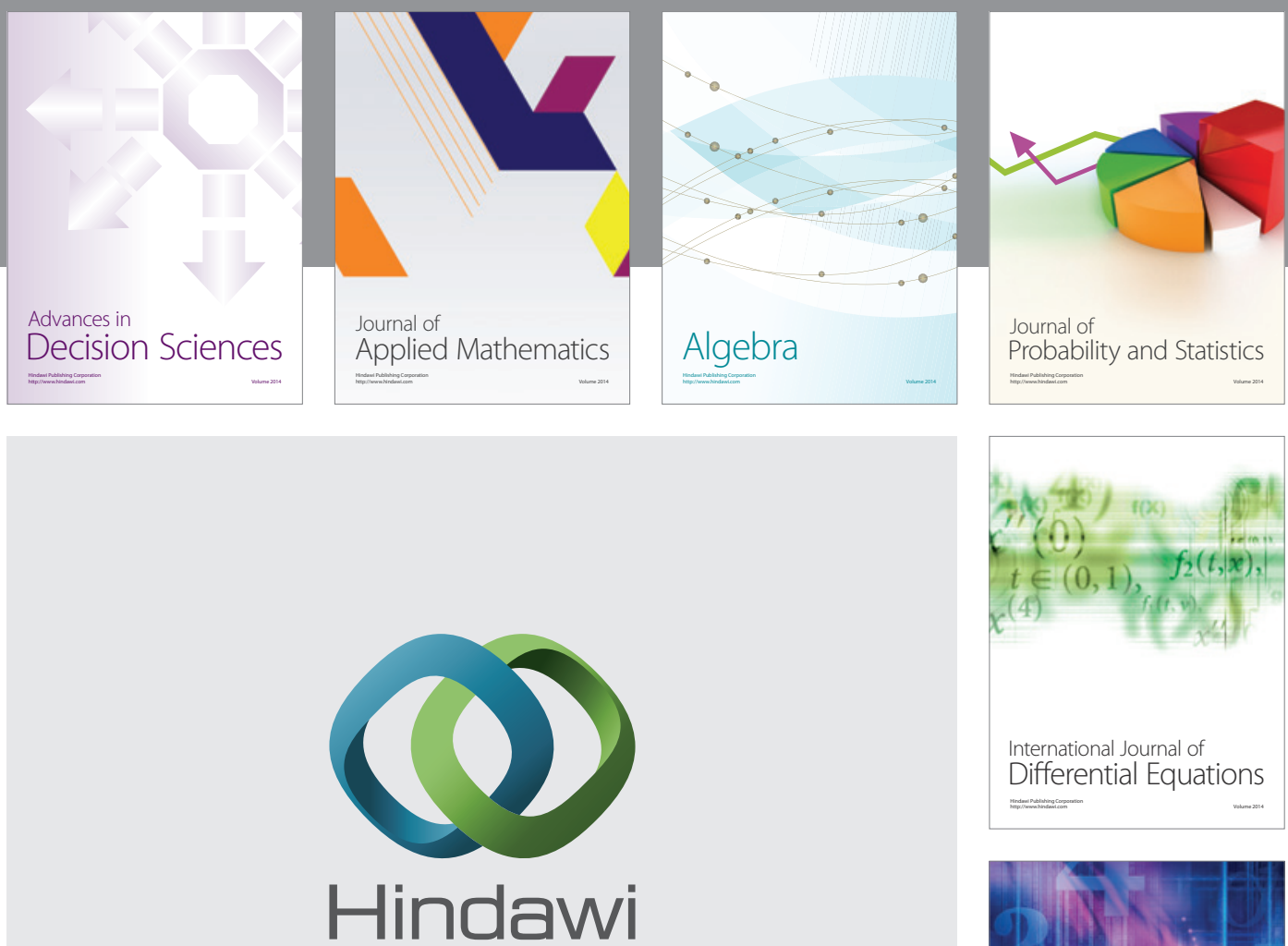

Submit your manuscripts at http://www.hindawi.com
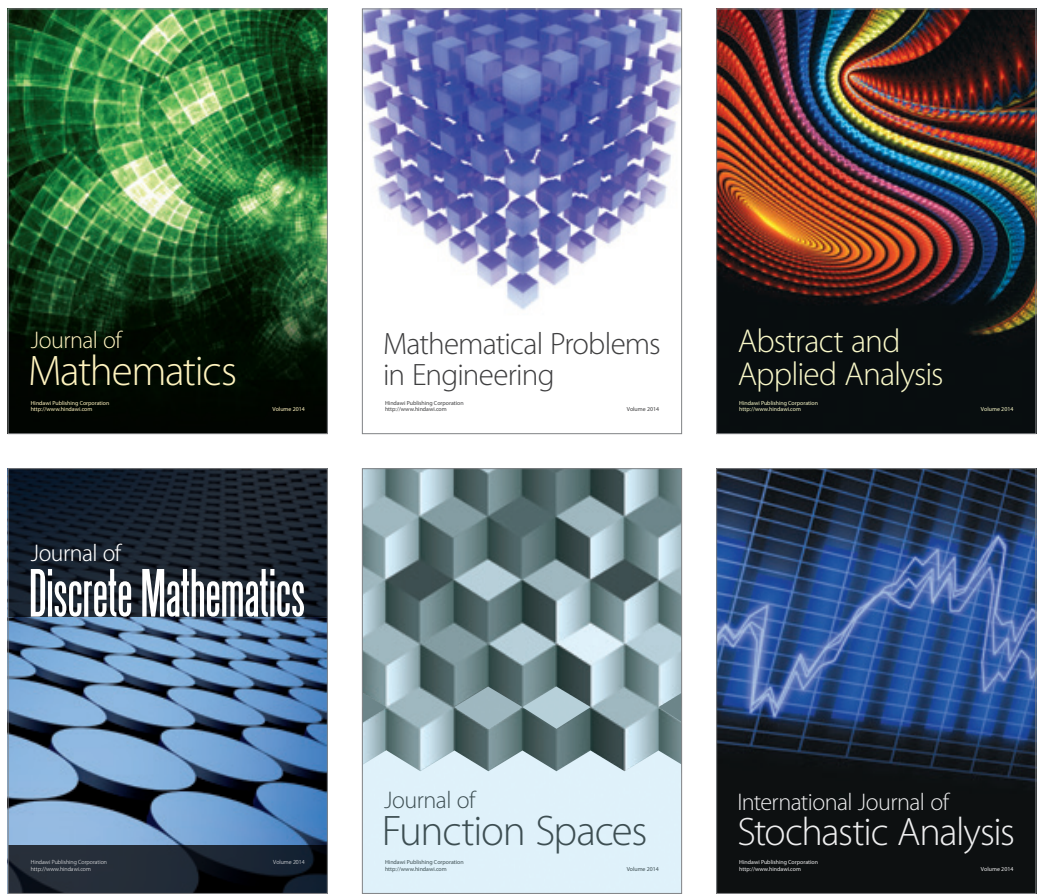

Journal of

Function Spaces

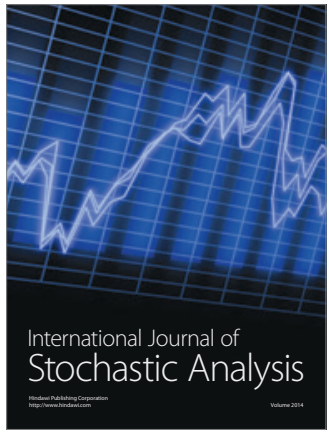

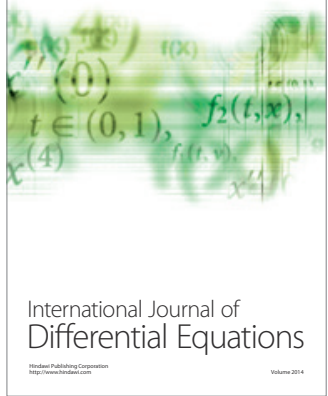
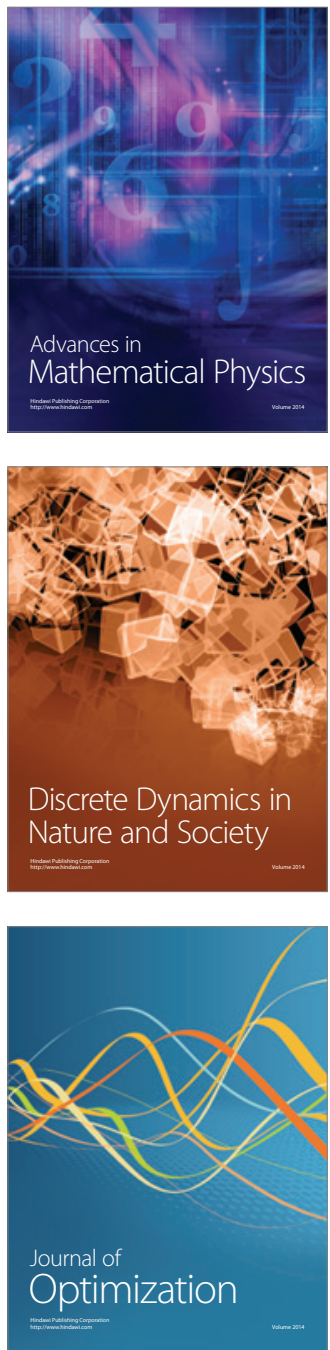